\title{
Average distances on self-similar sets and higher order average distances of self-similar measures
}

\author{
D. Allen ${ }^{1} \cdot$ H. Edwards ${ }^{2} \cdot$ S. Harper ${ }^{3} \cdot$ L. Olsen ${ }^{4}$
}

Received: 28 August 2015 / Accepted: 1 November 2016 / Published online: 29 December 2016

(C) The Author(s) 2016. This article is published with open access at Springerlink.com

\begin{abstract}
The purpose of this paper is twofold: (1) we study different notions of the average distance between two points of a self-similar subset of $\mathbb{R}$, and (2) we investigate the asymptotic behaviour of higher order average moments of self-similar measures on self-similar subsets of $\mathbb{R}$.
\end{abstract}

Keywords Self-similar set $\cdot$ Self-similar measures $\cdot$ Average distance $\cdot$ Moments $\cdot$ Mellin transform

Mathematics Subject Classification $28 \mathrm{~A} 78 \cdot 28 \mathrm{~A} 80$

\section{Introduction}

The purpose of this paper is twofold, namely, to study the average distance between two points of a self-similar subset of $\mathbb{R}$ and to investigate the asymptotic behaviour of higher order

L. Olsen

lo@st-and.ac.uk

D. Allen

dda505@york.ac.uk

H. Edwards

s1044536@sms.ed.ac.uk

S. Harper

scott.harper@bristol.ac.uk

1 Department of Mathematics, University of York, York YO10 5DD, England

2 School of Informatics, University of Edinburgh Appleton Tower, Edinburgh EH8 9LE, Scotland, UK

3 School of Mathematics, University of Bristol, Bristol BS8 1TW, England

4 Department of Mathematics, University of St. Andrews, St. Andrews, Fife KY16 9SS, Scotland, UK 
average moments of self-similar measures in $\mathbb{R}$. In particular, we investigate the following two problems:

(1) We compute the "natural geometric" average distance between two points in a selfsimilar Cantor subset $C$ of $\mathbb{R}$ satisfying the so-called Open Set Condition. If $C_{k}$ denotes the $k$ 'th order approximation to $C$ (the precise definition of $C_{k}$ will be given in Sect. 1.1), then the number

$$
\frac{\int_{C_{k}^{2}}|x-y| d(x, y)}{\int_{C_{k}^{2}} d(x, y)},
$$

may be interpreted as the average distance between two points chosen uniformly from $C_{k}$. We now show that the following limiting average distance, namely,

$$
\lim _{k} \frac{\int_{C_{k}^{2}}|x-y| d(x, y)}{\int_{C_{k}^{2}} d(x, y)}
$$

exists and we provide an explicit value for it; this is the content of Corollary 2.2 (we note that this result is not new, but was first proved by Leary et al. [18] in 2010; see below for a more detailed discussion of this).

There is a another, and perhaps equally natural, way to define the average distance between two points from $C$. Namely, the average distance between two points in $C$ chosen with respect to the "natural" uniform distribution on $C$, i.e. chosen with respect to the normalised Hausdorff measure on $C$. More precisely, if $s$ denotes the Hausdorff dimension of $C$ and $\mathcal{H}^{s}$ denotes the $s$-dimensional Hausdorff measure, then we compute the average distance between two points in $C$ chosen with respect to the normalised $s$-dimensional Hausdorff measure on $C$, i.e. we compute the integral

$$
\frac{1}{\mathcal{H}^{s}(C)^{2}} \int_{C^{2}}|x-y| d\left(\mathcal{H}^{s} \times \mathcal{H}^{s}\right)(x, y)
$$

this is the content of Corollary 2.3. Somewhat surprisingly, in general, the averages in (1.1) and (1.2) do not coincide. For example, if $C$ denotes the self-similar subset of $\mathbb{R}$ such that $C=S_{1} C \cup S_{2} C$ where $S_{1}, S_{2}:[0,1] \rightarrow[0,1]$ are defined by $S_{1}(x)=\frac{1}{4} x$ and $S_{2}(x)=$ $\frac{1}{2} x+\frac{1}{2}$, then

$$
\lim _{k} \frac{\int_{C_{k}^{2}}|x-y| d(x, y)}{\int_{C_{k}^{2}} d(x, y)}=\frac{8}{21} \approx 0.381
$$

and

$$
\frac{1}{\mathcal{H}^{s}(C)^{2}} \int_{C^{2}}|x-y| d\left(\mathcal{H}^{s} \times \mathcal{H}^{s}\right)(x, y)=\frac{12}{5(4+\sqrt{5})} \approx 0.385
$$

see Sect. 2.1.

In fact, we compute far more general averages than those in (1.1) and (1.2). Namely, if $\mu_{\mathbf{p}}$ and $\mu_{\mathbf{q}}$ are self-similar measures on $C$ associated with the probability vectors $\mathbf{p}$ and $\mathbf{q}$ (the precise definitions will be given in Sect. 1.1), then we compute the average distance between two points in $C$ where the first point is chosen with respect to the measure $\mu_{\mathbf{p}}$ and where the second point is chosen with respect to the measure $\mu_{\mathbf{q}}$, i.e. we compute the average distance defined by

$$
\int_{C^{2}}|x-y| d\left(\mu_{\mathbf{p}} \times \mu_{\mathbf{q}}\right)(x, y)
$$


see Theorem 2.1. For special choices of the probability vectors $\mathbf{p}$ and $\mathbf{q}$, the average in (1.3) simplifies to (1.1) and (1.2). Namely, if $C$ is generated by the Iterated Function System $\left(S_{1}, \ldots, S_{N}\right)$ where each $S_{i}:[0,1] \rightarrow[0,1]$ is a similarity map such that $S_{i}(0,1) \cap$ $S_{j}(0,1)=\varnothing$ for $i \neq j$ and the contracting ratio of $S_{i}$ is denoted to $r_{i}$ (the precise definitions of these concepts will be given in Sect. 1.1), then (1.3) simplifies to (1.1) for $\mathbf{p}=\mathbf{q}=$ $\left(\frac{r_{1}}{\sum_{i} r_{i}}, \ldots, \frac{r_{N}}{\sum_{i} r_{i}}\right)$, and (1.3) simplifies to (1.2) for $\mathbf{p}=\mathbf{q}=\left(r_{1}^{s}, \ldots, r_{N}^{s}\right)$ where $s$ is the unique solution to the equation $\sum_{i} r_{i}^{s}=1$.

The average distance between two points in a self-similar subset of $\mathbb{R}$ has recently been investigated by Leary et al. [18] and Bailey et al. [1]. In particular, Leary et al. proved the formula in Corollary 2.2 for the limiting "geometric" average distance in (1.1). Leary et al also provide formulas for the average distance (1.1) between points belonging to some selfsimilar subsets of $\mathbb{R}^{n}$ for $n \geq 2$, and for points belonging to certain families of fat Cantor subsets of $\mathbb{R}$. Averages similar to (1.1) between points belonging to self-similar subsets of $\mathbb{R}^{n}$, have also been studied in Bailey et al. [1]. In particular, Bailey et al. are interested in developing numerical methods that allow for high-precision approximation of the integrals in (1.1). Finally, we note that other notions of average distances on fractals (different from the ones considered in this paper and in [1,18]) have been studied by Bandt and Kuschel [2] and Hinz and Schief [14].

There is also a connection between the results in this paper and recent studies of the Kantorovich-Wasserstein distance between two self-similar measures. We first recall the definition of the Kantorovich-Wasserstein distance $W(\mu, v)$ between two Borel probability measures $\mu$ and $\nu$ on a compact metric space $X$. We say that a Borel probability measure $\gamma$ on $X \times X$ is a coupling of $\mu$ and $\nu$ if $\mu=\gamma \circ P^{-1}$ and $\nu=\gamma \circ Q^{-1}$ where $P, Q: X \times X \rightarrow X$ are the projections given by $P(x, y)=x$ and $Q(x, y)=y$, and we denote the family of couplings of $\mu$ and $v$ by $\Gamma(\mu, v)$. The Kantorovich-Wasserstein distance $W(\mu, v)$ between $\mu$ and $v$ is now defined by

$$
W(\mu, v)=\inf _{\gamma \in \Gamma(\mu, v)} \int|x-y| d \gamma(x, y),
$$

see, for example [20]. It is well-known that convergence with respect to the KantorovichWasserstein distance is equivalent to weak convergence. The Kantorovich-Wasserstein distance between self-similar measures has recently been studied by Fraser [11] and investigated further by Cipriano and Pollicott [4] and Cipriano [5]. In particular, Fraser [11] found an explicit formula for the Kantorovich-Wasserstein distance between two self-similar measures on the real line generated by Iterated Function Systems of two maps with a common contractions ratio. For the self-similar measures $\mu_{\mathbf{p}}$ and $\mu_{\mathbf{q}}$, the product measure $\mu_{\mathbf{p}} \times \mu_{\mathbf{q}}$ is clearly a coupling of $\mu_{\mathbf{p}}$ and $\mu_{\mathbf{q}}$ (but typically not a coupling that realises the KantorovichWasserstein distance between $\mu_{\mathbf{p}}$ and $\mu_{\mathbf{q}}$ ), and our results are therefore related to the study of the Kantorovich-Wasserstein distance between self-similar measures in [4,5,11]. For example, in order to derive his main results, Fraser [11, Theorem 2.1] first finds an explicit formula for the average $\int|x-y| d \gamma_{r}(x, y)$ for a certain family of self-similar measures $\gamma_{r}$ indexed by a parameter $r$, and a special case of this result is a special case of Theorem 2.1 providing an explicit formula for the average $\int|x-y| d\left(\mu_{\mathbf{p}} \times \mu_{\mathbf{q}}\right)(x, y)$.

(2) We find the exact asymptotic behaviour of the higher order average moments of a selfsimilar measure on a self-similar subset of $\mathbb{R}$; this is the contents of Theorems 2.4 and 2.5. More precisely, if $C$ is a self-similar subset of $\mathbb{R}$ generated by an Iterated Function System of the form $\left(S_{1}, S_{2}\right)$ where 


$$
\begin{aligned}
& S_{1}(x)=r x, \\
& S_{2}(x)=r x+1-r,
\end{aligned}
$$

for $r \in\left(0, \frac{1}{2}\right]$, i.e. $C$ is the unique non-empty compact subset of $\mathbb{R}$ such that

$$
C=S_{1} C \cup S_{2} C \text {. }
$$

(the precise definitions are given in Sect. 1.1) and $\mu_{\mathbf{p}}$ is the self-similar measure on $C$ associated with the probability vector $\mathbf{p}=\left(p_{1}, p_{2}\right)$, then we establish the exact asymptotic behaviour of the higher order moments

$$
M_{n}=\int_{C^{2}}|x-y|^{n} d\left(\mu_{\mathbf{p}} \times \mu_{\mathbf{p}}\right)(x, y)
$$

as $n$ tends to infinity. In particular, in Theorems 2.4 and 2.5 we show that there is a multiplicatively periodic function $\Pi:(0, \infty) \rightarrow \mathbb{R}$ with period equal to $r$ and a sequence $\left(\varepsilon_{n}\right)_{n}$ of real numbers with $\varepsilon_{n} \rightarrow 0$, such that

$$
n^{\Delta} M_{n}=\Pi(n)+\varepsilon_{n}
$$

for all $n$, where

$$
\Delta=\frac{\log p_{1} p_{2}}{\log r} .
$$

The proofs of Theorems 2.4 and 2.5 use techniques from number theory and dynamical systems involving Tauberian theory and "zeta-functions". Using a Tauberian argument (namely, the Mellin transform theorem), we first show that the $n$ 'th moment $M_{n}$ can be written as the sum of a complex contour integral of an appropriate "zeta-function" and an error-term. Next, we compute the complex contour integral using the residue theorem. In particular, we show that the contour integral can be written as the sum of a multiplicatively periodic function $\Pi(n)$ of $n$ and another error-term. Finally, combining these two results leads to (1.5).

We are, of course, not the first to investigate the asymptotic behaviour of different types of moments. In particular, the moments $J_{n}$ defined below have been studied, namely, if $C$ is a self-similar subset of the unit interval satisfying the Open Set Condition and $\mu_{\mathbf{p}}$ is the self-similar measure on $C$ associated with the probability vector $\mathbf{p}$, then the $n$ 'th moment $J_{n}$ is defined by

$$
J_{n}=\int_{C} t^{n} d \mu_{\mathbf{p}}(t)
$$

for $n \in \mathbb{N}$. For example, within the past 15 years several authors $[1,7,8]$ have outlined arguments suggesting that Tauberian theory and "zeta-functions" can be used to investigate the asymptotic behaviour of the moments $J_{n}$ and related quantities of special classes of self-similar measures and developed numerical methods that allow for high-precision approximations.

More rigorous studies of special cases of the above constructions have also been considered. For example, Cristea and Prodinger [6] and Grabner and Prodinger [13] have outlined how the same techniques, involving Tauberian theory and "zeta-functions", can be used to study the asymptotic behaviour of the moments $J_{n}$ of so-called binomial measures, i.e. the measures obtained by putting $r=\frac{1}{2}$ and $\mathbf{p}=\left(p_{1}, p_{2}\right)$, and Goh \& Wimp [12] use Tauberian theory to study the asymptotic behaviour of the moments $J_{n}$ of the Cantor measure, i.e. the measure obtained by putting $r=\frac{1}{3}$ and $\mathbf{p}=\left(p_{1}, p_{2}\right)=\left(\frac{1}{2}, \frac{1}{2}\right)$, providing full and rigorous proofs of their results. 
More recently Mellin transform ideas and "zeta-functions" have been introduced into fractal geometry by Lapidus et al. [16,17]. In particular, due to work by Lapidus and his collaborators $[16,17]$, it has now been recognized that the study of different types of moments is deeply related to the understanding of the geometry of many fractal sets and measures. This viewpoint is also illustrated by the following observation. Namely, the drop-off rate $\Delta$ of the moments $M_{n}$ equals the sum of the local dimension $\operatorname{dim}_{\text {loc }}\left(\mu_{\mathbf{p}} ; 0\right)=\frac{\log p_{1}}{\log r}$ of $\mu_{\mathbf{p}}$ at 0 and the local dimension $\operatorname{dim}_{\text {loc }}\left(\mu_{\mathbf{p}} ; 1\right)=\frac{\log p_{2}}{\log r}$ of $\mu_{\mathbf{p}}$ at 1, i.e.

$$
\Delta=\frac{\log p_{1} p_{2}}{\log r}=\frac{\log p_{1}}{\log r}+\frac{\log p_{2}}{\log r}=\operatorname{dim}_{\mathrm{loc}}\left(\mu_{\mathbf{p}} ; 0\right)+\operatorname{dim}_{\mathrm{loc}}\left(\mu_{\mathbf{p}} ; 1\right) ;
$$

recall, that if $\mu$ is a Borel measure on $\mathbb{R}^{n}$ and $x \in \mathbb{R}^{n}$, then the local dimension of $\mu$ at $x$ is defined by

$$
\operatorname{dim}_{\mathrm{loc}}(\mu ; x)=\lim _{\delta \searrow 0} \frac{\log \mu(B(x, \delta))}{\log \delta}
$$

provided the limit exists, see, for example $[9,10]$.

\subsection{The setting: self-similar sets and self-similar measures in $\mathbb{R}$}

Let $N$ be a positive integer with $N>1$, and fix real positive numbers $a_{1}, \ldots, a_{N}$ and $r_{1}, \ldots r_{N}$, with

$$
0=a_{1}<a_{1}+r_{1} \leq a_{2}<a_{2}+r_{2} \leq \cdots \leq a_{N}<a_{N}+r_{N}=1 .
$$

Define $S_{i}:[0,1] \rightarrow[0,1]$, by

$$
S_{i}(x)=r_{i} x+a_{i}
$$

It follows (see, for example [9]) that there is a unique non-empty compact subset $C$ of $[0,1]$ such that

$$
C=\bigcup_{i} S_{i} C
$$

the set $C$ is known as the self-similar set associated with the list $\left(S_{i}\right)_{i}$. The set $C$ can also be constructed as follows. In order to describe this construction, we introduce the following notation. For a positive integer $n$, write

$$
\Sigma^{n}=\{1, \ldots, N\}^{n}
$$

and

$$
\Sigma^{\mathbb{N}}=\{1, \ldots, N\}^{\mathbb{N}},
$$

i.e. $\Sigma^{n}=\{1, \ldots, N\}^{n}$ denotes the family of strings $\mathbf{i}=i_{1} \ldots i_{n}$ of length $n$ with $i_{j} \in$ $\{1, \ldots, N\}$ for all $j$ and $\Sigma^{\mathbb{N}}=\{1, \ldots, N\}^{\mathbb{N}}$ denotes the family of infinitely long strings $\mathbf{i}=i_{1} i_{2} \ldots$ with $i_{j} \in\{1, \ldots, N\}$ for all $j$. For $\mathbf{i}=i_{1} \ldots i_{n} \in \Sigma^{n}$, we will write $|\mathbf{i}|=n$ for the length of $\mathbf{i}$. The set $C$ can now be constructed as follows. For a positive integer $n$ and $\mathbf{i}=i_{1} \ldots i_{n} \in \Sigma^{n}$, let

$$
I_{\mathbf{i}}=S_{i_{1}} \ldots S_{i_{n}}[0,1]
$$


and

$$
C_{n}=\bigcup_{|\mathbf{i}|=n} I_{\mathbf{i}}
$$

Then $C_{0} \supseteq C_{1} \supseteq C_{2} \supseteq \cdots$ and $C$ equals the intersection of the $C_{n}$ 's, i.e.

$$
C=\bigcap_{n} C_{n}
$$

Loosely speaking (1.13) says that the $C_{n}$ may be thought of as approximations to the set $C$; this interpretation will be useful in Sect. 1.3.

In this paper, we will consider average distances (and higher order average moments) with respect to self-similar measures on $C$. Self-similar measures have attracted an enormous interest in the literature during the past 30 years and are defined as follows. Let $\mathbf{p}=\left(p_{1}, \ldots, p_{N}\right)$ be a probability vector. Then there is a unique Borel probability measure $\mu_{\mathbf{p}}$ supported on the self-similar set $C$ defined in (1.9) [or equivalently in (1.14)] such that

$$
\mu_{\mathbf{p}}=\sum_{i} p_{i} \mu_{\mathbf{p}} \circ S_{i}^{-1},
$$

the measure $\mu_{\mathbf{p}}$ is known as the self-similar measure (or the self-similar multifractal) associated with the list $\left(S_{i}, p_{i}\right)_{i}$, see, for example [10].

\subsection{Average distances and average moments: the measure theoretic approach}

For two Borel probability measures $\mu$ and $v$ on $C$, we define the average distance with respect to the measures $\mu$ and $v$ by

$$
A(\mu, v)=\int_{C^{2}}|x-y| d(\mu \times v)(x, y) .
$$

We are also interested in higher order average moments defined as follows. Namely, for a positive integer $n$, we define the $n$ 'th order average moment (or the $n$ 'th order average distance) with respect to the measures $\mu$ and $\nu$ by

$$
A^{n}(\mu, v)=\int_{C^{2}}|x-y|^{n} d(\mu \times v)(x, y) .
$$

\subsection{Average distances and average moments: the geometric approach}

There is a (perhaps) more intuitive approach for defining average distances and higher order average moments. In order to introduce this approach, we first introduce the following notation. If $\mu$ is a Borel measure on $\mathbb{R}$ and $E$ is a Borel subset of $\mathbb{R}$, then we write $\mu\llcorner E$ for the restriction of $\mu$ to $E$, i.e.

$$
(\mu\llcorner E)(B)=\mu(E \cap B)
$$

for any Borel subset $B$ of $\mathbb{R}$. Also, for $\mathbf{i}, \mathbf{j} \in \Sigma^{k}$, write $\lambda_{\mathbf{i}, \mathbf{j}}^{2}$ for the normalized 2-dimensional Lebesgue measure restricted to $I_{\mathbf{i}} \times I_{\mathbf{j}}$, i.e.

$$
\lambda_{\mathbf{i}, \mathbf{j}}^{2}=\frac{1}{r_{\mathbf{i}} r_{\mathbf{j}}} \mathcal{L}^{2}\left\llcorner\left(I_{\mathbf{i}} \times I_{\mathbf{j}}\right)\right.
$$

where $\mathcal{L}^{2}$ denotes the 2-dimensional Lebesgue measure. 
We can now describe the alternative (and perhaps) more intuitive geometric approach for defining average distances and higher order average moments. For Borel probability measures $\mu$ and $v$ on $C$ and a positive integer $k$, we define the $k$ 'th approximative $n$ 'th order average moment with respect to $\mu$ and $v$ by

$$
\begin{aligned}
A_{\mathrm{geo}, k}^{n}(\mu, v) & =\int_{C_{k}^{2}}|x-y|^{n} d\left(\sum_{|\mathbf{i}|=|\mathbf{j}|=k} \mu\left(I_{\mathbf{i}}\right) v\left(I_{\mathbf{j}}\right) \lambda_{\mathbf{i}, \mathbf{j}}^{2}\right)(x, y) \\
& =\sum_{|\mathbf{i}|=|\mathbf{j}|=k} \frac{\mu\left(I_{\mathbf{i}}\right) v\left(I_{\mathbf{j}}\right)}{r_{\mathbf{i}} r_{\mathbf{j}}} \int_{I_{\mathbf{i}} \times I_{\mathbf{j}}}|x-y|^{n} d(x, y) .
\end{aligned}
$$

Finally, we define the geometric average $n$ 'th order moment with respect to $\mu$ and $v$ by

$$
A_{\mathrm{geo}}^{n}(\mu, v)=\lim _{k} A_{\mathrm{geo}, k}^{n}(\mu, v),
$$

provided the limit exists. If $n=1$, then we will write $A_{\text {geo, } k}(\mu, v)$ and $A_{\text {geo }}(\mu, v)$ for $A_{\text {geo }, k}^{1}(\mu, v)$ and $A_{\text {geo }}^{1}(\mu, v)$, respectively.

The number $A_{\text {geo, }, k}(\mu, v)$ has a clear geometric interpretation. Namely, two players A and $\mathrm{B}$, say, throw darts at the $k$ 'th approximation $C_{k}=\cup_{|\mathbf{i}|=k} I_{\mathbf{i}}$ to the Cantor set $C$. If for each $\mathbf{i} \in \Sigma^{k}$, we make the following two assumptions, namely:

Assumption 1 Player A has the probability $\mu\left(I_{\mathbf{i}}\right)$ of hitting $I_{\mathbf{i}}$.

Assumption 2 Player B has the probability $v\left(I_{\mathbf{i}}\right)$ of hitting $I_{\mathbf{i}}$.

then the number $A_{\text {geo, },}(\mu, v)$ is the average distance between a dart thrown by $\mathrm{A}$ and a dart thrown by $\mathrm{B}$; of course, this game of darts is most likely not very realistic since the distribution of someone throwing darts at a line is more likely to be Gaussian than modelled by the measures $\mu$ and $\nu$.

The next result shows that this approach leads to the same notion of average distance as the measure theoretical approach in (1.17); more precisely, the result shows that the limit $A_{\text {geo }}^{n}(\mu, v)=\lim _{k} A_{\text {geo }, k}^{n}(\mu, v)$ always exists and equals $A^{n}(\mu, v)$.

Proposition 1.1 Let $\mu$ and $\nu$ be non-atomic Borel probability measures on $C$ and let $n$ be a positive integer. Then the limit $A_{\mathrm{geo}}^{n}(\mu, \nu)$ exists and

$$
A_{\text {geo }}^{n}(\mu, v)=A^{n}(\mu, v) .
$$

Proof For $\mathbf{i} \in \Sigma^{n}$, let $\lambda_{\mathbf{i}}^{1}$ denote the normalized Lebesgue measure restricted to $I_{\mathbf{i}}$, i.e. $\lambda_{\mathbf{i}}^{1}=\frac{1}{r_{\mathbf{i}}} \mathcal{L}^{1}\left\llcorner I_{\mathbf{i}}\right.$ where $\mathcal{L}^{1}$ denotes the 1-dimensional Lebesgue measure. Next, for a positive integer $k$, define measures $\tilde{\mu}_{k}$ and $\tilde{v}_{k}$ by $\tilde{\mu}_{k}=\sum_{|\mathbf{i}|=k} \mu\left(I_{\mathbf{i}}\right) \lambda_{\mathbf{i}}^{1}$ and $\tilde{v}_{k}=\sum_{|\mathbf{i}|=k} v\left(I_{\mathbf{i}}\right) \lambda_{\mathbf{i}}^{1}$. Since $\mu$ and $v$ are non-atomic, it is not difficult to see that $\tilde{\mu}_{k} \rightarrow \mu$ weakly and that $\tilde{v}_{k} \rightarrow v$ weakly, and it therefore follows from [3, Section 3.4] that $\tilde{\mu}_{k} \times \tilde{v}_{k} \rightarrow \mu \times \nu$. In particular, since clearly $A_{\text {geo }, k}^{n}(\mu, v)=\int|x-y|^{n} d\left(\tilde{\mu}_{k} \times \tilde{v}_{k}\right)(x, y)$ and $A^{n}(\mu, v)=\int|x-y|^{n} d(\mu \times v)(x, y)$, this now implies that

$A_{\mathrm{geo}, k}^{n}(\mu, v)=\int_{C_{k}^{2}}|x-y|^{n} d\left(\tilde{\mu}_{k} \times \tilde{v}_{k}\right)(x, y) \rightarrow \int_{C^{2}}|x-y|^{n} d(\mu \times v)(x, y)=A^{n}(\mu, v)$.

This completes the proof. 


\section{Statements of results}

\subsection{First order moments}

We first compute the average distance $A\left(\mu_{\mathbf{p}}, \mu_{\mathbf{q}}\right)$ with respect to two self-similar measures $\mu_{\mathbf{p}}$ and $\mu_{\mathbf{q}}$ associated with two (not necessarily identical) probability vectors $\mathbf{p}$ and $\mathbf{q}$; this is the content of the next theorem. Below we use the following notation, namely, for $i, j=$ $1, \ldots, N$, we write $s_{i, j}$ for the sign of $i-j$, i.e.

$$
s_{i, j}= \begin{cases}\frac{i-j}{|i-j|} & \text { for } i \neq j \\ 0 & \text { for } i=j\end{cases}
$$

Theorem 2.1 Let $\mathbf{p}=\left(p_{1}, \ldots, p_{N}\right)$ and $\mathbf{q}=\left(q_{1}, \ldots, q_{N}\right)$ be probability vectors. Then we have

$$
\begin{aligned}
A\left(\mu_{\mathbf{p}}, \mu_{\mathbf{q}}\right)= & A_{\text {geo }}\left(\mu_{\mathbf{p}}, \mu_{\mathbf{q}}\right) \\
= & \frac{1}{1-\sum_{i} p_{i} q_{i} r_{i}}\left(\sum_{i, j} p_{i} q_{j}\left|a_{i}-a_{j}\right|+\frac{\sum_{i} p_{i} a_{i}}{1-\sum_{i} p_{i} r_{i}} \sum_{i, j} s_{i, j} p_{i} q_{j} r_{i}\right. \\
& \left.+\frac{\sum_{i} q_{i} a_{i}}{1-\sum_{i} q_{i} r_{i}} \sum_{i, j} s_{i, j} p_{j} q_{i} r_{i}\right) .
\end{aligned}
$$

The proof of Theorem 2.1 is given in Sect. 3. We remark that the proof of Theorem 2.1 is not difficult. Indeed, we first derive a 1'st order linear difference equation for the sequence $\left(A_{\text {geo }, k}\left(\mu_{\mathbf{p}}, \mu_{\mathbf{q}}\right)\right)_{k}$. Using standard methods, this equation can now be solved giving the limiting behaviour of $A_{\text {geo }, k}\left(\mu_{\mathbf{p}}, \mu_{\mathbf{q}}\right)$ as $k \rightarrow \infty$.

If $\mathbf{p}=\mathbf{q}$ and all the contraction ratios coincide, i.e. if $r_{1}=\cdots=r_{N}=r$, then the formula in Theorem 2.1 for the average $A\left(\mu_{\mathbf{p}}, \mu_{\mathbf{q}}\right)$ simplifies considerably, namely, in this case it is easily seen that

$$
A\left(\mu_{\mathbf{p}}, \mu_{\mathbf{p}}\right)=A_{\mathrm{geo}}\left(\mu_{\mathbf{p}}, \mu_{\mathbf{p}}\right)=\frac{\sum_{i, j} p_{i} p_{j}\left|a_{i}-a_{j}\right|}{1-r \sum_{i} p_{i}^{2}} .
$$

Below we consider two corollaries of Theorem 2.1. By applying Theorem 2.1 to the vectors $\mathbf{p}=\mathbf{q}=\mathbf{u}$ where $\mathbf{u}=\left(\frac{r_{1}}{S}, \ldots, \frac{r_{N}}{S}\right)$ and $S=\sum_{i} r_{i}$, we obtain the first corollary, i.e. Corollary 2.2. This corollary shows that the following natural geometric limiting average distance, namely, $\lim _{k} \frac{\int_{C_{k}^{2}}|x-y| d(x, y)}{\int_{C_{k}^{2}} d(x, y)}$, exists and provides an explicit value for it. This result was first obtained by Leary et al. [18] in 2010.

Corollary 2.2 [18] We have

$$
\begin{aligned}
\lim _{k} \frac{\int_{C_{k}^{2}}|x-y| d(x, y)}{\int_{C_{k}^{2}} d(x, y)}= & \frac{1}{\left(\sum_{i} r_{i}\right)^{2}-\sum_{i} r_{i}^{3}}\left(\sum_{i, j} r_{i} r_{j}\left|a_{i}-a_{j}\right|\right. \\
& \left.+2 \frac{\sum_{i} r_{i} a_{i}}{\sum_{i} r_{i}-\sum_{i} r_{i}^{2}} \sum_{i, j} s_{i, j} r_{i}^{2} r_{j}\right) .
\end{aligned}
$$


Proof Define the probability vector $\mathbf{u}$ by $\mathbf{u}=\left(\frac{r_{1}}{S}, \ldots, \frac{r_{N}}{S}\right)$ where $S=\sum_{i} r_{i}$. It is clear that

$$
A_{\mathrm{geo}, k}\left(\mu_{\mathbf{u}}, \mu_{\mathbf{u}}\right)=\frac{\int_{C_{k}^{2}}|x-y| d(x, y)}{\int_{C_{k}^{2}} d(x, y)},
$$

and the result therefore follows immediately from Theorem 2.1 .

The second corollary, i.e. Corollary 2.3, computes the average distance between two points in $C$ with respect to the natural uniform distribution on $C$, namely, the normalised Hausdorff measure. To state this formally, we introduce the following notation. For a positive number $t$, let $\mathcal{H}^{t}$ denote the $t$-dimensional Hausdorff measure. Corollary 2.3 now gives an explicit value for the average distance between two points in $C$ with respect to the normalised Hausdorff measure, i.e. $\frac{1}{\mathcal{H}^{s}(C)^{2}} \int_{C^{2}}|x-y| d\left(\mathcal{H}^{s} \times \mathcal{H}^{s}\right)(x, y)$ where $s$ denotes the Hausdorff dimension of $C$.

Corollary 2.3 Let $s$ denote the Hausdorff dimension of $C$, i.e. $s$ is the unique real number such that $\sum_{i} r_{i}^{s}=1$ (see [9]). Then we have

$$
\begin{aligned}
\frac{1}{\mathcal{H}^{s}(C)^{2}} \int_{C^{2}}|x-y| d\left(\mathcal{H}^{s} \times \mathcal{H}^{s}\right)(x, y)= & \frac{1}{1-\sum_{i} r_{i}^{2 s+1}}\left(\sum_{i, j} r_{i}^{s} r_{j}^{s}\left|a_{i}-a_{j}\right|\right. \\
& \left.+2 \frac{\sum_{i} r_{i}^{s} a_{i}}{1-\sum_{i} r_{i}^{s+1}} \sum_{i, j} s_{i, j} r_{i}^{s+1} r_{j}^{s}\right) .
\end{aligned}
$$

Proof Since $\sum_{i} r_{i}^{s}=1$, we can define the probability vector $\mathbf{h}$ by $\mathbf{h}=\left(r_{1}^{s}, \ldots, r_{N}^{s}\right)$. It is well-known that the measure $\mu_{\mathbf{h}}$ equals the normalised $s$-dimensional Hausdorff measure on $C$, i.e. $\mu_{\mathbf{h}}=\frac{1}{\mathcal{H}^{s}(C)} \mathcal{H}^{s}\llcorner C$ (see, for example $[9,10]$ ), whence

$$
A\left(\mu_{\mathbf{h}}, \mu_{\mathbf{h}}\right)=\frac{1}{\mathcal{H}^{s}(C)^{2}} \int_{C^{2}}|x-y| d\left(\mathcal{H}^{s} \times \mathcal{H}^{s}\right)(x, y),
$$

and the result therefore follows immediately from Theorem 2.1 .

If all the contraction ratios coincide, i.e. if $r_{1}=\cdots=r_{N}=r$, then it is easily seen that the two "natural" averages in (2.2) and (2.3) coincide and that their common value equals $\frac{\sum_{i, j}\left|a_{i}-a_{j}\right|}{N^{2}-N r}$, i.e.

$$
\lim _{k} \frac{\int_{C_{k}^{2}}|x-y| d(x, y)}{\int_{C_{k}^{2}} d(x, y)}=\frac{1}{\mathcal{H}^{s}(C)^{2}} \int_{C^{2}}|x-y| d\left(\mathcal{H}^{s} \times \mathcal{H}^{s}\right)(x, y)=\frac{\sum_{i, j}\left|a_{i}-a_{j}\right|}{N^{2}-N r} .
$$

However, it is interesting to note that the two "natural" averages in (2.2) and (2.3) do not, in general, coincide. For example, let $C$ denote the self-similar set obtained by letting $N=2$, $r_{1}=\frac{1}{4}, r_{2}=\frac{1}{2}, a_{1}=0$ and $a_{2}=\frac{1}{2}$. It follows easily from Corollary 2.2 that in this case $\lim _{k} \frac{\int_{C_{k}^{2}}|x-y| d(x, y)}{\int_{C_{k}^{2}} d(x, y)}=\frac{8}{21} \approx 0.381$. Also, the Hausdorff dimension $s$ of $C$ satisfies the equation $r_{1}^{s}+r_{2}^{s}=1$, and so $s=\frac{\log \varphi}{\log 2}$ where $\varphi=\frac{1+\sqrt{5}}{2}$ is the golden ratio, whence $r_{1}^{s}=\varphi^{-2}$ and $r_{2}^{s}=\varphi^{-1}$. Using this (and the fact that $\varphi^{2}+\varphi=1$ ), it now follows from Corollary 2.3 that $\frac{1}{\mathcal{H}^{s}(C)^{2}} \int_{C^{2}}|x-y| d\left(\mathcal{H}^{s} \times \mathcal{H}^{s}\right)(x, y)=\frac{12}{5(4+\sqrt{5})} \approx 0.385$. 


\subsection{Higher order moments}

The second main result in this paper establishes the exact asymptotic behaviour of the average moments $A^{n}\left(\mu_{\mathbf{p}}, \mu_{\mathbf{q}}\right)$ as $n \rightarrow \infty$ in the special, but important, case when $\mathbf{p}=\mathbf{q}$ and all of the contracting ratios $r_{i}$ coincide. More precisely, in this section we will assume that $N=2$ and that the contraction ratios $r_{1}$ and $r_{2}$ are equal and we will denote the common value by $r$, i.e.

$$
r_{1}=r_{2}=r .
$$

Also, let $\mathbf{p}=\left(p_{1}, p_{2}\right)$ be a probability vector and write

$$
p=p_{1}^{2}+p_{2}^{2}, \quad q=p_{1} p_{2} .
$$

Finally, let

$$
\Delta=\frac{\log q}{\log r} .
$$

We will now analyse the moments $A^{n}\left(\mu_{\mathbf{p}}, \mu_{\mathbf{p}}\right)$. It is not difficult to find a recursive formula for the moments $A^{n}\left(\mu_{\mathbf{p}}, \mu_{\mathbf{p}}\right)$. Indeed, in Lemma 4.3 we prove that

$$
A^{n}\left(\mu_{\mathbf{p}}, \mu_{\mathbf{p}}\right)=p r^{n} A^{n}\left(\mu_{\mathbf{p}}, \mu_{\mathbf{p}}\right)+2 q \sum_{i=0}^{\left[\frac{n}{2}\right]}\left(\begin{array}{c}
n \\
2 i
\end{array}\right)(1-r)^{n-2 i} r^{2 i} A^{2 i}\left(\mu_{\mathbf{p}}, \mu_{\mathbf{p}}\right),
$$

where $\left[\frac{n}{2}\right]$ denotes the integer part of the real number $\frac{n}{2}$. While the above recursive formula provides an expression for $A^{n}\left(\mu_{\mathbf{p}}, \mu_{\mathbf{p}}\right)$, this expression is not easy to analyse. For this reason, it seems more meaningful to find explicit formulas describing the asymptotic behaviour of $A^{n}\left(\mu_{\mathbf{p}}, \mu_{\mathbf{p}}\right)$ for large $n$. We first note that it is clear that $A^{n}\left(\mu_{\mathbf{p}}, \mu_{\mathbf{p}}\right) \rightarrow 0$ as $n \rightarrow \infty$, and it is therefore interesting and natural to ask how fast $A^{n}\left(\mu_{\mathbf{p}}, \mu_{\mathbf{p}}\right)$ tends to 0 as $n \rightarrow \infty$. We answer this question in Theorem 2.5. In particular, we prove that

$$
\frac{\log A^{n}\left(\mu_{\mathbf{p}}, \mu_{\mathbf{p}}\right)}{\log n} \rightarrow-\Delta
$$

this result clearly provides information about how fast $A^{n}\left(\mu_{\mathbf{p}}, \mu_{\mathbf{p}}\right)$ tends to 0 as $n \rightarrow \infty$. Indeed loosely speaking (2.6) says that:

$$
A^{n}\left(\mu_{\mathbf{p}}, \mu_{\mathbf{p}}\right) \text { behaves like } \frac{1}{n^{\Delta}} \text { for large } n \text {. }
$$

In fact, Theorem 2.5 provides significantly more detailed information. Not only does Theorem 2.5 show that $A^{n}\left(\mu_{\mathbf{p}}, \mu_{\mathbf{p}}\right)$ behaves like $\frac{1}{n^{\Delta}}$ for large $n$, but it gives an exact and explicit asymptotic expression for $A^{n}\left(\mu_{\mathbf{p}}, \mu_{\mathbf{p}}\right)$, namely, it shows that $n^{\Delta} A^{n}\left(\mu_{\mathbf{p}}, \mu_{\mathbf{p}}\right)$ equals a multiplicatively period function of $n$ plus an error term that tends to 0 as $n \rightarrow \infty$.

Theorem 2.4 There is a function $\Pi:(0, \infty) \rightarrow \mathbb{C}$ and a sequence $\left(\varepsilon_{n}\right)_{n}$ of real numbers satisfying the following two conditions:

(1) $\Pi$ is multiplicatively periodic with period equal to $r$, i.e. $\Pi(r u)=\Pi(u)$ for all $u$;

(2) $\lim \sup _{n} n\left|\varepsilon_{n}\right|<\infty$; in particular $\varepsilon_{n} \rightarrow 0$,

such that

$$
n^{\Delta} A^{n}\left(\mu_{\mathbf{p}}, \mu_{\mathbf{p}}\right)=\Pi(n)+\varepsilon_{n}
$$


for all $n$. In particular,

$$
\frac{\log A^{n}\left(\mu_{\mathbf{p}}, \mu_{\mathbf{p}}\right)}{\log n} \rightarrow-\Delta .
$$

In fact, our methods allow us to obtain an explicit expression for the periodic function $\Pi$. This is the contents of the next theorem. In Theorem 2.5 we use the following notation, namely, we write $[x]$ for the integer part of a real number $x$.

Theorem 2.5 Define the sequence $\left(\lambda_{k}\right)_{k}$ recursively by

$$
\lambda_{0}=1 \quad \lambda_{k}=p r^{k} \lambda_{k}+2 q \sum_{i=0}^{\left[\frac{k}{2}\right]}\left(\begin{array}{c}
k \\
2 i
\end{array}\right)(1-r)^{k-2 i} r^{2 i} \lambda_{2 i} .
$$

Then the series $\sum_{k=0}^{\infty} \frac{\lambda_{k}}{k !}(r s)^{k}\left(p e^{-s}+q(-1)^{k} e^{-r s}\right)$ converges for all $s \in \mathbb{C}$, and we can define the function $\Lambda: \mathbb{C} \rightarrow \mathbb{C}$ by

$$
\Lambda(s)=\sum_{k=0}^{\infty} \frac{\lambda_{k}}{k !}(r s)^{k}\left(p e^{-s}+q(-1)^{k} e^{-r s}\right) .
$$

Then $\int_{0}^{\infty}\left|\Lambda(u) u^{s-1}\right| d u<\infty$ for $s \in \mathbb{C}$ with $\operatorname{Re} s>0$, and we can define $Z:\{s \in$ $\mathbb{C} \mid \operatorname{Re} s>0\} \rightarrow \mathbb{C}$ by

$$
Z(s)=\int_{0}^{\infty} \Lambda(u) u^{s-1} d u
$$

For $n \in \mathbb{Z}$, write $s_{n}=\Delta+\frac{1}{-\log r} 2 \pi \dot{\mathrm{i}}$. Then the trigonometric series $\sum_{n \in \mathbb{Z}} Z\left(s_{n}\right) e^{2 \pi \dot{\mathrm{i}} \frac{\log u}{\log r}}$ converges for all $u>0$, and

$$
\Pi(u)=\frac{1}{-\log r} \sum_{n \in \mathbb{Z}} Z\left(s_{n}\right) e^{2 \pi \dot{\mathrm{i}} \frac{\log u}{\log r}}
$$

for $u>0$.

The proofs of Theorems 2.4 and 2.5 are given in Sects. 4, 5, 6 and 7. The proofs are divided into three parts. To briefly describe this, we introduce the following notation, namely, write $M_{n}=A^{n}\left(\mu_{\mathbf{p}}, \mu_{\mathbf{p}}\right)$ and define $L: \mathbb{C} \rightarrow \mathbb{C}$ by

$$
L(s)=\sum_{n=0}^{\infty} \frac{M_{n}}{n !} s^{n} e^{-s} .
$$

Section 4 contains a number of useful technical estimates of the auxiliary function $L$ and the function $\Lambda$ in Theorem 2.5. The remaining part of the proofs are now divided into the following three parts.

Part 1, Sect. 5 We first show that there is constant $K$ such that

$$
\left|A^{n}\left(\mu_{\mathbf{p}}, \mu_{\mathbf{p}}\right)-L(n)\right| \leq K \frac{1}{n^{\Delta+1}}
$$

for all $n$. The proof of (2.7) follows from Cauchy's formula applied to the function $L$ and is presented in Sect. 5. 
Part 2, Sect. 6 Next, we show that for each real number $d$ with $d>\Delta$ there is a constant $K_{d}$ such that

$$
\left|L(u)-u^{-\Delta} \Pi(u)\right| \leq K_{d} \frac{1}{u^{d}}
$$

for all $u>0$ where $\Pi$ is the function defined in Theorem 2.5. The proof of (2.8) is presented in Sect. 6 and is divided in the following two sub-parts:

Part 2.1 Using the Mellin transform theory, we show that $L$ can be written as a complex curve integral involving $Z$, namely, we show that for $0<c<\Delta$ we have

$$
L(u)=\frac{1}{2 \pi \dot{\mathrm{i}}} \int_{c-\dot{\mathrm{i}} \infty}^{c+\dot{\mathrm{i}} \infty} \frac{Z(s)}{1-q r^{-s}} u^{-s} d s
$$

for all $u>0$; this is done in Theorem 6.3.

Part 2.2 Next, using the residue theorem, we compute the complex curve integral in (2.9). In particular, we show that if $0<c<\Delta<d$, then

$$
\frac{1}{2 \pi \dot{\mathrm{i}}} \int_{c-\mathrm{i} \infty}^{c+\mathrm{i} \infty} \frac{Z(s)}{1-q r^{-s}} u^{-s} d s=u^{-\Delta} \Pi(u)+\mathcal{O}\left(\frac{1}{u^{d}}\right)
$$

for all $u>0$; this is done in Theorems 6.4, 6.5 and 6.6: in Theorems 6.4 and 6.5 estimates for $|Z(s)|$ and $\left|1-q r^{-s}\right|$ are obtained and in Theorem 6.6 we use the residue theorem together with the estimates from Theorems 6.4 and 6.5 to derive formula (2.10).

The desired inequality [i.e. (2.8)] follows immediately from combining (2.9) and (2.10). Part 3, Sect. 7 Finally, Theorems 2.4 and 2.5 follow by combining (2.7) and (2.8). This is done in Sect. 7.

\section{Proof of Theorem 2.1}

For brevity, we write

$$
A_{k}=A_{\text {geo }, k}\left(\mu_{\mathbf{p}}, \mu_{\mathbf{q}}\right)=\sum_{|\mathbf{i}|=|\mathbf{j}|=k} \frac{p_{\mathbf{i}} q_{\mathbf{j}}}{r_{\mathbf{i}} r_{\mathbf{j}}} \int_{I_{\mathbf{i}} \times I_{\mathbf{j}}}|x-y| d(x, y) .
$$

for positive integers $k$. Our aim now is to find an explicit formula for $\lim _{k} A_{k}$; observe that it follows from Proposition 1.1 that the $\operatorname{limit}_{\lim } A_{k}$ exists. We first introduce the following notation. For a probability vector $\pi=\left(\pi_{1}, \ldots, \pi_{N}\right)$ and a positive integer $k$ and $\mathbf{i}=i_{1} \ldots i_{k} \in \Sigma^{k}$, we write $\pi_{\mathbf{i}}=\pi_{i_{1}} \ldots \pi_{i_{k}}$ and

$$
\begin{aligned}
B_{\pi, k} & =\sum_{|\mathbf{i}|=k} \frac{\pi_{\mathbf{i}}}{r_{\mathbf{i}}} \int_{I_{\mathbf{i}}} t d t, \\
B_{\pi} & =\frac{\sum_{i} \pi_{i} a_{i}}{1-\sum_{i} \pi_{i} r_{i}} .
\end{aligned}
$$

Below we show that the elements in the sequence $\left(A_{k}\right)_{k}$ satisfy a recursive formula involving the $B_{\mathbf{p}, k}$ 's and the $B_{\mathbf{q}, k}$ 's. The limiting behaviour of the $A_{k}$ 's can then be established from this recursive formula using the following well-known (and easily proven) result.

Lemma 3.1 Let $t \in \mathbb{R}$ with $|t|<1$ and let $\left(y_{n}\right)_{n}$ be a sequence of real numbers such that $y_{n} \rightarrow y$. Let the sequence $\left(x_{n}\right)_{n}$ be defined by

$$
x_{n+1}=t x_{n}+y_{n}
$$


for all $n$. Then

$$
x_{n} \rightarrow \frac{y}{1-t}
$$

We will now obtain recursive formulas for the $B_{\pi, k}$ 's and the $A_{k}$ 's; this is done in Propositions 3.2 and 3.3 below.

Proposition 3.2 Let $\pi=\left(\pi_{1}, \ldots, \pi_{N}\right)$ be a probability vector.

(1) For all positive integers $k$, we have $B_{\pi, k+1}=\left(\sum_{i} \pi_{i} r_{i}\right) B_{\pi, k}+\sum_{i} \pi_{i} a_{i}$.

(2) We have $B_{\pi, k} \rightarrow B_{\pi}$.

Proof (1) For all positive integers $k$, we have

$$
B_{\pi, k+1}=\sum_{i} \sum_{|\mathbf{i}|=k} \frac{\pi_{\mathbf{i}}}{r_{\mathbf{i}}} \frac{\pi_{i}}{r_{i}} \int_{I_{i \mathbf{i}}} t d t .
$$

However, it is clear that if $\mathbf{i} \in \Sigma^{k}$ and $i=1, \ldots, N$, then $I_{i \mathbf{i}}=S_{i} I_{\mathbf{i}}$, whence $\int_{I_{i \mathbf{i}}} t d t=$ $\int_{S i I_{\mathbf{i}}} t d t=\int_{I_{\mathbf{i}}} S_{i}(u) S_{i}^{\prime}(u) d u=\int_{I_{\mathbf{i}}}\left(r_{i} u+a_{i}\right) r_{i} d u$, and it therefore follows from (3.4) that

$$
\begin{aligned}
B_{\pi, k} & =\sum_{i} \sum_{|\mathbf{i}|=k} \frac{\pi_{\mathbf{i}}}{r_{\mathbf{i}}} \frac{\pi_{i}}{r_{i}} \int_{I_{\mathbf{i}}}\left(r_{i} u+a_{i}\right) r_{i} d u \\
& =\left(\sum_{i} \pi_{i} r_{i}\right)\left(\sum_{|\mathbf{i}|=k} \frac{\pi_{\mathbf{i}}}{r_{\mathbf{i}}} \int_{I_{\mathbf{i}}} u d u\right)+\left(\sum_{i} \pi_{i} a_{i}\right)\left(\sum_{|\mathbf{i}|=k} \frac{\pi_{\mathbf{i}}}{r_{\mathbf{i}}} \int_{I_{\mathbf{i}}} d u\right) .
\end{aligned}
$$

Using the fact that $\sum_{|\mathbf{i}|=k} \frac{\pi_{\mathbf{i}}}{r_{\mathbf{i}}} \int_{I_{\mathbf{i}}} u d u=B_{\pi, k}$ and $\sum_{|\mathbf{i}|=k} \frac{\pi_{\mathbf{i}}}{r_{\mathbf{i}}} \int_{I_{\mathbf{i}}} d u=\sum_{|\mathbf{i}|=k} \frac{\pi_{\mathbf{i}}}{r_{\mathbf{i}}} r_{\mathbf{i}}=$ $\sum_{|\mathbf{i}|=k} \pi_{\mathbf{i}}=1$, we now deduce from (3.5) that

$$
B_{\pi, n+1}=\left(\sum_{i} \pi_{i} r_{i}\right) B_{\pi, n}+\sum_{i} \pi_{i} a_{i} .
$$

(2) This statement follows immediately from Part (1) and Lemma 3.1.

Proposition 3.3 For $i, j=1, \ldots, N$, recall that $s_{i, j}$ denotes the sign of $i-j$, and for a positive integer $k$ write

$$
Y_{k}=\sum_{i, j} p_{i} q_{j}\left|a_{i}-a_{j}\right|+B_{\mathbf{p}, k} \sum_{i, j} s_{i, j} p_{i} q_{j} r_{i}+B_{\mathbf{q}, k} \sum_{i, j} s_{i, j} p_{j} q_{i} r_{i} .
$$

(1) For all positive integers $k$, we have $A_{k+1}=\left(\sum_{i} p_{i} q_{i} r_{i}\right) A_{k}+Y_{k}$.

(2) We have $Y_{k} \rightarrow \sum_{i, j} p_{i} q_{j}\left|a_{i}-a_{j}\right|+B_{\mathbf{p}} \sum_{i, j} s_{i, j} p_{i} q_{j} r_{i}+B_{\mathbf{q}} \sum_{i, j} s_{i, j} p_{j} q_{i} r_{i}$.

Proof (1) For all positive integers $k$, we have

$$
A_{k+1}=\sum_{i, j} \sum_{|\mathbf{i}|=|\mathbf{j}|=k} \frac{p_{i} q_{j}}{r_{i} r_{j}} \frac{p_{\mathbf{i}} q_{\mathbf{j}}}{r_{\mathbf{i}} r_{\mathbf{j}}} \int_{I_{i \mathbf{i}} \times I_{j \mathbf{j}}}|x-y| d(x, y) .
$$

However, it is clear that if $\mathbf{i}, \mathbf{j} \in \Sigma^{k}$ and $i, j=1, \ldots, N$, then $I_{i \mathbf{i}}=S_{i} I_{\mathbf{i}}$ and $I_{j \mathbf{j}}=S_{j} I_{\mathbf{j}}$, whence $\int_{I_{i \mathbf{i}} \times I_{j \mathbf{j}}}|x-y| d(x, y)=\int_{S_{i} I_{\mathbf{i}} \times S_{j} I_{\mathbf{j}}}|x-y| d(x, y)=\int_{I_{\mathbf{i}} \times I_{\mathbf{j}}} \mid S_{i}(u)-$ 
$S_{j}(v)\left|r_{i} r_{j} d(u, v)=\int_{I_{\mathbf{i}} \times I_{\mathbf{j}}}\right|\left(r_{i} u+a_{i}\right)-\left(r_{j} v+a_{j}\right) \mid r_{i} r_{j} d(u, v)$, and it therefore follows from (3.6) that

$$
\begin{aligned}
A_{k+1}= & \sum_{i, j} \sum_{|\mathbf{i}|=|\mathbf{j}|=k} \frac{p_{i} q_{j}}{r_{i} r_{j}} \frac{p_{\mathbf{i}} q_{\mathbf{j}}}{r_{\mathbf{i}} r_{\mathbf{j}}} \int_{I_{\mathbf{i}} \times I_{\mathbf{j}}}\left|\left(r_{i} u+a_{i}\right)-\left(r_{j} v+a_{j}\right)\right| r_{i} r_{j} d(u, v) \\
= & \sum_{i=j} \sum_{|\mathbf{i}|=|\mathbf{j}|=k} p_{i} q_{j} \frac{p_{\mathbf{i}} q_{\mathbf{j}}}{r_{\mathbf{i}} r_{\mathbf{j}}} \int_{I_{\mathbf{i}} \times I_{\mathbf{j}}}\left|\left(r_{i} u+a_{i}\right)-\left(r_{j} v+a_{j}\right)\right| d(u, v) \\
& +\sum_{i \neq j} \sum_{|\mathbf{i}|=|\mathbf{j}|=k} p_{i} q_{j} \frac{p_{\mathbf{i}} q_{\mathbf{j}}}{r_{\mathbf{i}} r_{\mathbf{j}}} \int_{I_{\mathbf{i}} \times I_{\mathbf{j}}}\left|\left(r_{i} u+a_{i}\right)-\left(r_{j} v+a_{j}\right)\right| d(u, v) \\
= & \left(\sum_{i} p_{i} q_{i} r_{i}\right) \sum_{|\mathbf{i}|=|\mathbf{j}|=k} \frac{p_{\mathbf{i}} q_{\mathbf{j}}}{r_{\mathbf{i}} r_{\mathbf{j}}} \int_{I_{\mathbf{i}} \times I_{\mathbf{j}}}|u-v| d(u, v) \\
& +\sum_{i \neq j} \sum_{|\mathbf{i}|=|\mathbf{j}|=k} p_{i} q_{j} \frac{p_{\mathbf{i}} q_{\mathbf{j}}}{r_{\mathbf{i}} r_{\mathbf{j}}} \int_{I_{\mathbf{i}} \times I_{\mathbf{j}}}\left|\left(r_{i} u+a_{i}\right)-\left(r_{j} v+a_{j}\right)\right| d(u, v) .
\end{aligned}
$$

Next, since $r_{i} u+a_{i}=S_{i} u \in S_{i}([0,1])$ and $r_{j} v+a_{j}=S_{j} v \in S_{j}([0,1])$ for all $i, j=$ $1, \ldots, N$ and $u, v \in[0,1]$, we conclude that $\left|\left(r_{i} u+a_{i}\right)-\left(r_{j} v+a_{j}\right)\right|=s_{i, j}\left(\left(r_{i} u+a_{i}\right)-\right.$ $\left.\left(r_{j} v+a_{j}\right)\right)$ for all $i, j=1, \ldots, N$ with $i \neq j$ and $u, v \in[0,1]$. This and (3.7) imply that

$$
\begin{aligned}
A_{k+1}= & \left(\sum_{i} p_{i} q_{i} r_{i}\right) \sum_{|\mathbf{i}|=|\mathbf{j}|=k} \frac{p_{\mathbf{i}} q_{\mathbf{j}}}{r_{\mathbf{i}} r_{\mathbf{j}}} \int_{I_{\mathbf{i}} \times I_{\mathbf{j}}}|u-v| d(u, v) \\
& +\sum_{i \neq j} \sum_{|\mathbf{i}|=|\mathbf{j}|=k} p_{i} q_{j} \frac{p_{\mathbf{i}} q_{\mathbf{j}}}{r_{\mathbf{i}} r_{\mathbf{j}}} \int_{I_{\mathbf{i}} \times I_{\mathbf{j}}} s_{i, j}\left(\left(r_{i} u+a_{i}\right)-\left(r_{j} v+a_{j}\right)\right) d(u, v) \\
= & \left(\sum_{i} p_{i} q_{i} r_{i}\right) \sum_{|\mathbf{i}|=|\mathbf{j}|=n} \frac{p_{\mathbf{i}} q_{\mathbf{j}}}{r_{\mathbf{i}} r_{\mathbf{j}}} \int_{I_{\mathbf{i}} \times I_{\mathbf{j}}}|u-v| d(u, v) \\
& +\sum_{i \neq j} \sum_{|\mathbf{i}|=|\mathbf{j}|=k} \frac{p_{\mathbf{i}} q_{\mathbf{j}}}{r_{\mathbf{i}} r_{\mathbf{j}}} s_{i, j} p_{i} q_{j}\left(a_{i}-a_{j}\right) \int_{I_{\mathbf{i}} \times I_{\mathbf{j}}} d(u, v) \\
& +\sum_{i \neq j} \sum_{|\mathbf{i}|=|\mathbf{j}|=k} \frac{p_{\mathbf{i}} q_{\mathbf{j}}}{r_{\mathbf{i}} r_{\mathbf{j}}} s_{i, j} p_{i} q_{j} \int_{I_{\mathbf{i}} \times I_{\mathbf{j}}}\left(r_{i} u-r_{j} v\right) d(u, v) .
\end{aligned}
$$

Using the fact that $\sum_{|\mathbf{i}|=|\mathbf{j}|=k} \frac{p_{\mathbf{i}} q_{\mathbf{j}}}{r_{\mathbf{i}} r_{\mathbf{j}}} \int_{I_{\mathbf{i}} \times I_{\mathbf{j}}}|u-v| d(u, v)=A_{k}$ and $\sum_{i \neq j} s_{i, j} p_{i} q_{j}\left(a_{i}-\right.$ $\left.a_{j}\right) \int_{I_{\mathbf{i}} \times I_{\mathbf{j}}} d(u, v)=\sum_{i \neq j} p_{i} q_{j}\left|a_{i}-a_{j}\right| \int_{I_{\mathbf{i}} \times I_{\mathbf{j}}} d(u, v)=\sum_{i, j} p_{i} q_{j}\left|a_{i}-a_{j}\right|$ $\int_{I_{\mathbf{i}} \times I_{\mathbf{j}}} d(u, v)=\sum_{i, j} p_{i} q_{j}\left|a_{i}-a_{j}\right| r_{\mathbf{i}} r_{\mathbf{j}}$ (because $\left.s_{i, j}\left(a_{i}-a_{j}\right)=\left|a_{i}-a_{j}\right|\right)$, we conclude from (3.8) that

$$
\begin{aligned}
A_{k+1}= & \left(\sum_{i} p_{i} q_{i} r_{i}\right) A_{k}+\sum_{i, j} \sum_{|\mathbf{i}|=|\mathbf{j}|=k} p_{\mathbf{i}} q_{\mathbf{j}} p_{i} q_{j}\left|a_{i}-a_{j}\right| \\
& +\sum_{i \neq j} \sum_{|\mathbf{i}|=|\mathbf{j}|=k} \frac{p_{\mathbf{i}} q_{\mathbf{j}}}{r_{\mathbf{i}} r_{\mathbf{j}}} s_{i, j} p_{i} q_{j} \int_{I_{\mathbf{i}} \times I_{\mathbf{j}}}\left(r_{i} u-r_{j} v\right) d(u, v) .
\end{aligned}
$$


Since clearly $\sum_{|\mathbf{i}|=|\mathbf{j}|=k} p_{\mathbf{i}} q_{\mathbf{j}}=1$, this simplifies to

$$
\begin{aligned}
A_{k+1}= & \left(\sum_{i} p_{i} q_{i} r_{i}\right) A_{k}+\sum_{i, j} p_{i} q_{j}\left|a_{i}-a_{j}\right| \\
& +\sum_{i \neq j} \sum_{|\mathbf{i}|=|\mathbf{j}|=k} \frac{p_{\mathbf{i}} q_{\mathbf{j}}}{r_{\mathbf{i}} r_{\mathbf{j}}} s_{i, j} p_{i} q_{j} \int_{I_{\mathbf{i} \times I_{\mathbf{j}}}}\left(r_{i} u-r_{j} v\right) d(u, v) \\
= & \left(\sum_{i} p_{i} q_{i} r_{i}\right) A_{k}+\sum_{i, j} p_{i} q_{j}\left|a_{i}-a_{j}\right|+U_{k}
\end{aligned}
$$

where

$$
U_{k}=\sum_{i \neq j} \sum_{|\mathbf{i}|=|\mathbf{j}|=k} \frac{p_{\mathbf{i}} q_{\mathbf{j}}}{r_{\mathbf{i}} r_{\mathbf{j}}} s_{i, j} p_{i} q_{j} \int_{I_{\mathbf{i}} \times I_{\mathbf{j}}}\left(r_{i} u-r_{j} v\right) d(u, v) .
$$

We will now compute $U_{k}$. In particular, we will express $U_{k}$ in terms of $B_{\mathbf{p}, k}$ and $B_{\mathbf{q}, k}$. To do so we note that

$$
\begin{aligned}
U_{k}= & \sum_{i \neq j} s_{i, j} p_{i} q_{j} r_{i} \sum_{|\mathbf{i}|=|\mathbf{j}|=k} \frac{p_{\mathbf{i}} q_{\mathbf{j}}}{r_{\mathbf{i}} r_{\mathbf{j}}} \int_{I_{\mathbf{i}} \times I_{j}} u d(u, v) \\
& -\sum_{i \neq j} s_{i, j} p_{i} q_{j} r_{j} \sum_{|\mathbf{i}|=|\mathbf{j}|=k} \frac{p_{\mathbf{i}} q_{\mathbf{j}}}{r_{\mathbf{i}} r_{\mathbf{j}}} \int_{I_{\mathbf{i}} \times I_{j}} v d(u, v) \\
= & \sum_{i \neq j} s_{i, j} p_{i} q_{j} r_{i}\left(\sum_{|\mathbf{i}|=k} \frac{p_{\mathbf{i}}}{r_{\mathbf{i}}} \int_{I_{\mathbf{i}}} u d u\right)\left(\sum_{|\mathbf{j}|=k} \frac{q_{\mathbf{j}}}{r_{\mathbf{j}}} \int_{I_{\mathbf{j}}} d v\right) \\
& -\sum_{i \neq j} s_{i, j} p_{i} q_{j} r_{j}\left(\sum_{|\mathbf{i}|=k} \frac{p_{\mathbf{i}}}{r_{\mathbf{i}}} \int_{I_{\mathbf{i}}} d u\right)\left(\sum_{|\mathbf{j}|=k} \frac{q_{\mathbf{j}}}{r_{\mathbf{j}}} \int_{I_{\mathbf{j}}} v d v\right) .
\end{aligned}
$$

Using the fact that $\sum_{|\mathbf{i}|=k} \frac{p_{\mathbf{i}}}{r_{\mathbf{i}}} \int_{I_{\mathbf{i}}} u d u=B_{\mathbf{p}, k}, \sum_{|\mathbf{j}|=k} \frac{q_{\mathbf{j}}}{r_{\mathbf{j}}} \int_{I_{\mathbf{j}}} v d v=B_{\mathbf{q}, k}, \sum_{|\mathbf{i}|=k} \frac{p_{\mathbf{i}}}{r_{\mathbf{i}}} \int_{I_{\mathbf{i}}} d u=$ $\sum_{|\mathbf{i}|=k} \frac{p_{\mathbf{i}}}{r_{\mathbf{i}}} r_{\mathbf{i}}=\sum_{|\mathbf{i}|=k} p_{\mathbf{i}}=1$ and $\sum_{|\mathbf{j}|=k} \frac{q_{\mathbf{j}}}{r_{\mathbf{j}}} \int_{I_{\mathbf{j}}} d u=\sum_{|\mathbf{j}|=k} \frac{q_{\mathbf{j}}}{r_{\mathbf{j}}} r_{\mathbf{j}}=\sum_{|\mathbf{j}|=k} q_{\mathbf{j}}=1$, it follows from (3.10) that

$$
\begin{aligned}
U_{k} & =B_{\mathbf{p}, k} \sum_{i \neq j} s_{i, j} p_{i} q_{j} r_{i}-B_{\mathbf{q}, k} \sum_{i \neq j} s_{i, j} p_{i} q_{j} r_{j} \\
& =B_{\mathbf{p}, k} \sum_{i, j} s_{i, j} p_{i} q_{j} r_{i}-B_{\mathbf{q}, k} \sum_{i, j} s_{i, j} p_{i} q_{j} r_{j} \\
& =B_{\mathbf{p}, k} \sum_{i, j} s_{i, j} p_{i} q_{j} r_{i}+B_{\mathbf{q}, k} \sum_{i, j} s_{j, i} p_{i} q_{j} r_{j} .
\end{aligned}
$$

Finally, combining (3.9) and (3.11) shows that

$$
\begin{aligned}
A_{k+1} & =\left(\sum_{i} p_{i} q_{i} r_{i}\right) A_{k}+\sum_{i, j} p_{i} q_{j}\left|a_{i}-a_{j}\right|+B_{\mathbf{p}, k} \sum_{i, j} s_{i, j} p_{i} q_{j} r_{i}+B_{\mathbf{q}, k} \sum_{i, j} s_{j, i} p_{i} q_{j} r_{j} \\
& =\left(\sum_{i} p_{i} q_{i} r_{i}\right) A_{n}+Y_{n} .
\end{aligned}
$$


This completes the proof.

(2) This statement follows from Proposition 3.2.

We can now prove Theorem 2.1.

Proof of Theorem 2.1 It follows immediately from Lemma 3.1 and Proposition 3.3 that $A_{\text {geo }, k}\left(\mu_{\mathbf{p}}, \mu_{\mathbf{q}}\right)=A_{k} \rightarrow \frac{\sum_{i, j} p_{i} q_{j}\left|a_{i}-a_{j}\right|+B_{\mathbf{p}} \sum_{i, j} s_{i, j} p_{i} q_{j} r_{i}+B_{\mathbf{q}} \sum_{i, j} s_{i, j} p_{j} q_{i} r_{i}}{1-\sum_{i} p_{i} q_{i} r_{i}}$.

This completes the proof of Theorem 2.1.

\section{Proof of Theorems 2.4 and 2.5: the auxiliary functions $L$ and $\Lambda$}

The proofs of Theorems 2.4 and 2.5 are given in this and the next three sections. The main purpose of this section is to introduce the two key auxiliary functions $L$ and $\Lambda$, and to provide estimates for the derivatives and the integral of $\Lambda$; this is done in Propositions 4.5 and 4.6, respectively.

However, we first state and prove the following simple lemma that will be used several times in this and the next sections when estimating $L$ and $\Lambda$.

Lemma 4.1 Let $f, g: \mathbb{C} \rightarrow \mathbb{C}$ be functions and let $a, \rho$ be complex numbers with $|a|<1$ and $|\rho|<1$. Assume that

$$
f(s)=a f(\rho s)+g(s)
$$

for all $s \in \mathbb{C}$ and that $f$ is bounded in an open neighbourhood of 0 . Then the series $\sum_{k=0}^{\infty} a^{k} g\left(\rho^{k} s\right)$ converges for all $s \in \mathbb{C}$ and

$$
f(s)=\sum_{k=0}^{\infty} a^{k} g\left(\rho^{k} s\right)
$$

for $s \in \mathbb{C}$.

Proof Let $s$ be a complex number. Repeated use of (4.1) shows that $f(s)=a^{n} f\left(\rho^{n} s\right)+$ $\sum_{k=0}^{n} a^{k} g\left(\rho^{k} s\right)$ for all positive integers $n$, whence

$$
\left|f(s)-\sum_{k=0}^{n} a^{k} g\left(\rho^{k} s\right)\right| \leq|a|^{n}\left|f\left(\rho^{n} s\right)\right|
$$

for all positive integers $n$. Since $|a|<1$ and $|\rho|<1$, and $f$ is bounded in an open neighbourhood of 0 , it follows that $|a|^{n}\left|f\left(\rho^{n} s\right)\right| \rightarrow 0$ as $n \rightarrow \infty$, and we therefore deduce from (4.2) that $f(s)=\sum_{k \geq 0} a^{k} g\left(\rho^{k} s\right)$.

Next, we derive a recursive equation for the $n$ 'th moments $A_{\text {geo }, k}^{n}\left(\mu_{\mathbf{p}}, \mu_{\mathbf{p}}\right)$; this is done in Lemmas 4.2 and 4.3. The recursive equation in Lemma 4.3 plays a key role in proving the estimates in Propositions 4.5 and 4.6. For brevity we introduce the following notation. Namely, for non-negative integers $n$ and $k$, we write

$$
\begin{aligned}
M_{n, k} & =A_{\text {geo }, k}^{n}\left(\mu_{\mathbf{p}}, \mu_{\mathbf{p}}\right)=\sum_{|\mathbf{i}|=|\mathbf{j}|=k} \frac{p_{\mathbf{i}} p_{\mathbf{j}}}{r_{\mathbf{i}} r_{\mathbf{j}}} \int_{I_{\mathbf{i}} \times I_{\mathbf{j}}}|x-y|^{n} d(x, y), \\
M_{n} & =A^{n}\left(\mu_{\mathbf{p}}, \mu_{\mathbf{p}}\right)=\int_{C^{2}}|x-y|^{n} d\left(\mu_{\mathbf{p}} \times \mu_{\mathbf{p}}\right)(x, y) .
\end{aligned}
$$


Also, recall that $r_{1}=r_{2}=r$ and that $\mathbf{p}=\left(p_{1}, p_{2}\right)$. We also write

$$
p=p_{1}^{2}+p_{2}^{2}, \quad q=p_{1} p_{2} .
$$

Finally, we write $[x]$ for the integer part of a real number $x$.

Lemma 4.2 For all positive integers $n$ and $k$, we have

$$
M_{n, k+1}=p r^{n} M_{n, k}+2 q \sum_{i=0}^{\left[\frac{n}{2}\right]}\left(\begin{array}{c}
n \\
2 i
\end{array}\right)(1-r)^{n-2 i} r^{2 i} M_{2 i, k} .
$$

Proof For all positive integers $n$ and $k$, we have

$$
\begin{aligned}
M_{n, k+1} & =\sum_{i, j} \sum_{|\mathbf{i}|=|\mathbf{j}|=k} \frac{p_{i \mathbf{i}} p_{j \mathbf{j}}}{r_{i \mathbf{i}} r_{j \mathbf{j}}} \int_{I_{i \mathbf{i}} \times I_{j \mathbf{j}}}|x-y|^{n} d(x, y) \\
& =\sum_{i, j} \sum_{|\mathbf{i}|=|\mathbf{j}|=k} \frac{p_{i} p_{j}}{r_{i} r_{j}} \frac{p_{\mathbf{i}} p_{\mathbf{j}}}{r_{\mathbf{i}} r_{\mathbf{j}}} \int_{I_{i \mathbf{i}} \times I_{j \mathbf{j}}}|x-y|^{n} d(x, y) .
\end{aligned}
$$

However, it is clear that if $\mathbf{i}, \mathbf{j} \in \Sigma^{k}$ and $i, j=1, \ldots, N$, then $I_{i \mathbf{i}}=S_{i} I_{\mathbf{i}}$ and $I_{j \mathbf{j}}=S_{j} I_{\mathbf{j}}$, whence $\int_{I_{i \mathbf{i}} \times I_{j \mathbf{j}}}|x-y|^{n} d(x, y)=\int_{S_{i} I_{\mathbf{i}} \times S_{j} I_{\mathbf{j}}}|x-y|^{n} d(x, y)=\int_{I_{\mathbf{i}} \times I_{\mathbf{j}}} \mid S_{i}(u)-$ $\left.S_{j}(v)\right|^{n} r_{i} r_{j} d(u, v)=\int_{I_{\mathbf{i}} \times I_{\mathbf{j}}}\left|\left(r_{i} u+a_{i}\right)-\left(r_{j} v+a_{j}\right)\right|^{n} r_{i} r_{j} d(u, v)$, and it therefore follows from (4.3) that

$$
\begin{aligned}
M_{n, k+1}= & \sum_{i=j} \sum_{|\mathbf{i}|=|\mathbf{j}|=k} p_{i} p_{j} \frac{p_{\mathbf{i}} p_{\mathbf{j}}}{r_{\mathbf{i}} r_{\mathbf{j}}} \int_{I_{\mathbf{i}} \times I_{\mathbf{j}}}\left|\left(r_{i} u+a_{i}\right)-\left(r_{j} v+a_{j}\right)\right|^{n} d(u, v) \\
& +\sum_{i \neq j} \sum_{|\mathbf{i}|=|\mathbf{j}|=k} p_{i} p_{j} \frac{p_{\mathbf{i}} p_{\mathbf{j}}}{r_{\mathbf{i}} r_{\mathbf{j}}} \int_{I_{\mathbf{i}} \times I_{\mathbf{j}}}\left|\left(r_{i} u+a_{i}\right)-\left(r_{j} v+a_{j}\right)\right|^{n} d(u, v) \\
= & \left(\sum_{i} p_{i}^{2} r_{i}^{n}\right) \sum_{|\mathbf{i}|=|\mathbf{j}|=k} \frac{p_{\mathbf{i}} p_{\mathbf{j}}}{r_{\mathbf{i}} r_{\mathbf{j}}} \int_{I_{\mathbf{i}} \times I_{\mathbf{j}}}|u-v|^{n} d(u, v) \\
& +\sum_{i \neq j} \sum_{|\mathbf{i}|=|\mathbf{j}|=k} p_{i} p_{j} \frac{p_{\mathbf{i}} p_{\mathbf{j}}}{r_{\mathbf{i}} r_{\mathbf{j}}} \int_{I_{\mathbf{i}} \times I_{\mathbf{j}}}\left|\left(r_{i} u+a_{i}\right)-\left(r_{j} v+a_{j}\right)\right|^{n} d(u, v) .
\end{aligned}
$$

Using the fact that $\sum_{|\mathbf{i}|=|\mathbf{j}|=k} \frac{p_{\mathbf{i}} p_{\mathbf{j}}}{r_{\mathbf{i}} r_{\mathbf{j}}} \int_{I_{\mathbf{i}} \times I_{\mathbf{j}}}|u-v|^{n} d(u, v)=M_{n, k}$, (4.4) now simplifies to

$$
\begin{aligned}
M_{n, k+1}= & \left(\sum_{i} p_{i}^{2} r_{i}^{n}\right) M_{n . k}+\sum_{i \neq j} \sum_{|\mathbf{i}|=|\mathbf{j}|=k} p_{i} p_{j} \frac{p_{\mathbf{i}} p_{\mathbf{j}}}{r_{\mathbf{i}} r_{\mathbf{j}}} \int_{I_{\mathbf{i}} \times I_{\mathbf{j}}} \mid\left(r_{i} u+a_{i}\right) \\
& -\left.\left(r_{j} v+a_{j}\right)\right|^{n} d(u, v) .
\end{aligned}
$$

Next, since $r_{i} u+a_{i}=S_{i} u \in S_{i}([0,1])$ and $r_{j} v+a_{j}=S_{j} v \in S_{j}([0,1])$ for all $i, j=$ $1, \ldots, N$ and $u, v \in[0,1]$, we conclude that $\left|\left(r_{i} u+a_{i}\right)-\left(r_{j} v+a_{j}\right)\right|=s_{i, j}\left(\left(r_{i} u+a_{i}\right)-\right.$ $\left.\left(r_{j} v+a_{j}\right)\right)$ for all $i, j=1, \ldots, N$ with $i \neq j$ and $u, v \in[0,1]$. This and (4.5) imply that

$$
\begin{aligned}
M_{n, k+1}= & \left(\sum_{i} p_{i}^{2} r_{i}^{n}\right) M_{n . k}+\sum_{i \neq j} \sum_{|\mathbf{i}|=|\mathbf{j}|=k} p_{i} p_{j} \frac{p_{\mathbf{i}} p_{\mathbf{j}}}{r_{\mathbf{i}} r_{\mathbf{j}}} \int_{I_{\mathbf{i}} \times I_{\mathbf{j}}} s_{i, j}^{n}\left(\left(r_{i} u+a_{i}\right)\right. \\
& \left.-\left(r_{j} v+a_{j}\right)\right)^{n} d(u, v) \\
= & \left(\sum_{i} p_{i} p_{i} r_{i}^{n}\right) M_{n . k}+m_{n, k}
\end{aligned}
$$


where

$$
m_{n, k}=\sum_{i \neq j} \sum_{|\mathbf{i}|=|\mathbf{j}|=k} p_{i} p_{j} \frac{p_{\mathbf{i}} p_{\mathbf{j}}}{r_{\mathbf{i}} r_{\mathbf{j}}} \int_{I_{\mathbf{i}} \times I_{\mathbf{j}}} s_{i, j}^{n}\left(\left(r_{i} u+a_{i}\right)-\left(r_{j} v+a_{j}\right)\right)^{n} d(u, v) .
$$

We will now compute $m_{n, k}$. Since $i, j \in\{1,2\}, r_{1}=r_{2}=r, a_{1}=0, a_{2}=1-r$, $s_{1,2}=-1$ and $s_{2,1}=1$, we conclude that

$$
\begin{aligned}
m_{n, k}= & \sum_{|\mathbf{i}|=|\mathbf{j}|=k} p_{1} p_{2} \frac{p_{\mathbf{i}} p_{\mathbf{j}}}{r_{\mathbf{i}} r_{\mathbf{j}}} \int_{I_{\mathbf{i}} \times I_{\mathbf{j}}} s_{1,2}^{n}\left(\left(r u+a_{1}\right)-\left(r v+a_{2}\right)\right)^{n} d(u, v) \\
& +\sum_{|\mathbf{i}|=|\mathbf{j}|=k} p_{2} p_{1} \frac{p_{\mathbf{i}} p_{\mathbf{j}}}{r_{\mathbf{i}} r_{\mathbf{j}}} \int_{I_{\mathbf{i}} \times I_{\mathbf{j}}} s_{2,1}^{n}\left(\left(r u+a_{2}\right)-\left(r v+a_{1}\right)\right)^{n} d(u, v) \\
= & \sum_{|\mathbf{i}|=|\mathbf{j}|=k} p_{1} p_{2} \frac{p_{\mathbf{i}} p_{\mathbf{j}}}{r_{\mathbf{i}} r_{\mathbf{j}}} \int_{I_{\mathbf{i}} \times I_{\mathbf{j}}}(r(v-u)+(1-r))^{n} d(u, v) \\
& +\sum_{|\mathbf{i}|=|\mathbf{j}|=k} p_{1} p_{2} \frac{p_{\mathbf{i}} p_{\mathbf{j}}}{r_{\mathbf{i}} r_{\mathbf{j}}} \int_{I_{\mathbf{i}} \times I_{\mathbf{j}}}(r(u-v)+(1-r))^{n} d(u, v) \\
= & \sum_{|\mathbf{i}|=|\mathbf{j}|=k} p_{1} p_{2} \frac{p_{\mathbf{i}} p_{\mathbf{j}}}{r_{\mathbf{i}} r_{\mathbf{j}}} \int_{I_{\mathbf{i}} \times I_{\mathbf{j}}} \sum_{l=0}^{n}\left(\begin{array}{l}
n \\
l
\end{array}\right)(1-r)^{n-l} r^{l}(v-u)^{l} d(u, v) \\
& +\sum_{|\mathbf{i}|=|\mathbf{j}|=k} p_{1} p_{2} \frac{p_{\mathbf{i}} p_{\mathbf{j}}}{r_{\mathbf{i}} r_{\mathbf{j}}} \int_{I_{\mathbf{i}} \times I_{\mathbf{j}}} \sum_{l=0}^{n}\left(\begin{array}{l}
n \\
l
\end{array}\right)(1-r)^{n-l} r^{l}(u-v)^{l} d(u, v) \\
= & p_{1} p_{2} \sum_{l=0}^{n}\left(\begin{array}{l}
n \\
l
\end{array}\right)(1-r)^{n-l} r^{l} \sum_{|\mathbf{i}|=|\mathbf{j}|=k} \frac{p_{\mathbf{i}} p_{\mathbf{j}}}{r_{\mathbf{i}} r_{\mathbf{j}}} \int_{I_{\mathbf{i}} \times I_{\mathbf{j}}}\left((u-v)^{l}\right. \\
& \left.+(v-u)^{l}\right) d(u, v) .
\end{aligned}
$$

However, it is clear that if $(u, v) \in I_{\mathbf{i}} \times I_{\mathbf{j}}$ and $l$ is a positive integer, then $(u-v)^{l}+(v-u)^{l}=0$ if $l$ is odd and $(u-v)^{l}+(v-u)^{l}=2|u-v|^{l}$ if $l$ is even. It follows from this and (4.7) that

$$
\begin{aligned}
m_{n, k} & =2 p_{1} p_{2} \sum_{\substack{l=0, \ldots, n \\
l \text { is even }}}\left(\begin{array}{c}
n \\
l
\end{array}\right)(1-r)^{n-l} r^{l} \sum_{|\mathbf{i}|=|\mathbf{j}|=k} \frac{p_{\mathbf{i}} p_{\mathbf{j}}}{r_{\mathbf{i}} r_{\mathbf{j}}} \int_{I_{\mathbf{i}} \times I_{\mathbf{j}}}|u-v|^{l} d(u, v) \\
& =2 p_{1} p_{2} \sum_{i=0}^{\left[\frac{n}{2}\right]}\left(\begin{array}{c}
n \\
2 i
\end{array}\right)(1-r)^{n-2 i} r^{2 i} \sum_{|\mathbf{i}|=|\mathbf{j}|=k} \frac{p_{\mathbf{i}} p_{\mathbf{j}}}{r_{\mathbf{i}} r_{\mathbf{j}}} \int_{I_{\mathbf{i}} \times I_{\mathbf{j}}}|u-v|^{2 i} d(u, v) .
\end{aligned}
$$

Finally, using the fact that $\sum_{|\mathbf{i}|=|\mathbf{j}|=k} \frac{p_{\mathbf{i}} p_{\mathbf{j}}}{r_{\mathbf{i}} r_{\mathbf{j}}} \int_{I_{\mathbf{i}} \times I_{\mathbf{j}}}|u-v|^{2 i} d(u, v)=M_{2 i, k}$, the previous equality simplifies to

$$
m_{n, k}=2 p_{1} p_{2} \sum_{i=0}^{\left[\frac{n}{2}\right]}\left(\begin{array}{c}
n \\
2 i
\end{array}\right)(1-r)^{n-2 i} r^{2 i} M_{2 i, k}
$$

Combining (4.6) and (4.8) gives the desired result. 
Lemma 4.3 For all positive integers $n$, we have

$$
M_{n}=p r^{n} M_{n}+2 q \sum_{i=0}^{\left[\frac{n}{2}\right]}\left(\begin{array}{c}
n \\
2 i
\end{array}\right)(1-r)^{n-2 i} r^{2 i} M_{2 i}
$$

Proof Since $M_{n, k}=A_{\text {geo }, k}^{n}\left(\mu_{\mathbf{p}}, \mu_{\mathbf{p}}\right) \rightarrow A^{n}\left(\mu_{\mathbf{p}}, \mu_{\mathbf{p}}\right)=M_{n}$ for all $n$ (by Proposition 1.1), the statement follows immediately from Lemma 4.2.

We now turn towards the definitions of the auxiliary functions $L$ and $\Lambda$. We first define the moment generating function $M: \mathbb{C} \rightarrow \mathbb{C}$ by

$$
M(s)=\sum_{k=0}^{\infty} \frac{M_{k}}{k !} s^{k}
$$

for $s \in \mathbb{C}$; observe that since $\left|M_{k}\right|=\int|x-y|^{k} d\left(\mu_{\mathbf{p}} \times \mu_{\mathbf{p}}\right)(x, y) \leq 1$ for all $k$, it follows that the series $\sum_{n} \frac{M_{k}}{k !} s^{k}$ is convergent for all $s \in \mathbb{C}$. We also define the even and odd moment generating functions $M_{\mathrm{e}}, M_{\mathrm{\circ}}: \mathbb{C} \rightarrow \mathbb{C}$ by

$$
\begin{aligned}
& M_{\mathrm{e}}(s)=\sum_{k=0}^{\infty} \frac{M_{2 k}}{(2 k) !} s^{2 k}, \\
& M_{\mathrm{\circ}}(s)=\sum_{n=0}^{\infty} \frac{M_{2 k+1}}{(2 k+1) !} s^{2 k+1}
\end{aligned}
$$

for $s \in \mathbb{C}$; since $\left|M_{k}\right| \leq 1$ for all $k$, it follows that the series $\sum_{k=0}^{\infty} \frac{M_{2 k}}{(2 k) !} s^{2 k}$ and $\sum_{k=0}^{\infty} \frac{M_{2 k+1}}{(2 k+1) !} s^{2 k+1}$ converge for all $s \in \mathbb{C}$. Next, define $L, L_{\mathrm{e}}, L_{\mathrm{o}}: \mathbb{C} \rightarrow \mathbb{C}$ by

$$
\begin{aligned}
L(s) & =M(s) e^{-s}, \\
L_{\mathrm{e}}(s) & =M_{\mathrm{e}}(s) e^{-s}, \\
L_{\mathrm{o}}(s) & =M_{\mathrm{\circ}}(s) e^{-s}
\end{aligned}
$$

for $s \in \mathbb{C}$. Finally, define $\Lambda: \mathbb{C} \rightarrow \mathbb{C}$ by

$$
\Lambda(s)=p L(r s) e^{-(1-r) s}+q L(-r s) e^{-2 r s} .
$$

The next three results (i.e. Lemma 4.4, Propositions 4.5 and 4.6) are estimates involving the auxiliary functions $M, L$ and $\Lambda$; these results play key roles in the later sections of the paper. Lemma 4.4 provides functional equations for $M, M_{\mathrm{e}}$ and $M_{\mathrm{\circ}}$ and Propositions 4.5 and 4.6 provide estimates for the derivatives and the integral of $\Lambda$, respectively.

Lemma 4.4 (Functional equations for $M, M_{\mathrm{e}}$ and $M_{\mathrm{o}}$ ) For $s \in \mathbb{C}$, we have

$$
\begin{aligned}
M(s) & =p M(r s)+2 q M_{\mathrm{e}}(r s) e^{s(1-r)}, \\
M_{\mathrm{e}}(s) & =p M_{\mathrm{e}}(r s)+2 q \cosh (s(1-r)) M_{\mathrm{e}}(r s), \\
M_{\mathrm{\circ}}(s) & =p M_{\mathrm{\circ}}(r s)+2 q \sinh (s(1-r)) M_{\mathrm{e}}(r s) .
\end{aligned}
$$


Proof It follows from Lemma 4.3 and the definition of $M_{\mathrm{e}}(s)$ that

$$
\begin{aligned}
M_{\mathrm{e}}(s) & =\sum_{k=0}^{\infty} \frac{M_{2 k}}{(2 k) !} s^{2 k} \\
& =\sum_{k=0}^{\infty} \frac{1}{(2 k) !}\left(p r^{2 k} M_{2 k}+2 q \sum_{i=0}^{k}\left(\begin{array}{c}
2 k \\
2 i
\end{array}\right)(1-r)^{2 k-2 i} r^{2 i} M_{2 i}\right) s^{2 k} \\
& =p M_{\mathrm{e}}(r s)+2 q \sum_{k=0}^{\infty} \sum_{i=0}^{k} \frac{s^{2 k-2 i}}{(2 k-2 i) !}(1-r)^{2 k-2 i} \frac{s^{2 i}}{(2 i) !} r^{2 i} M_{2 i} \\
& =p M_{\mathrm{e}}(r s)+2 q\left(\sum_{k=0}^{\infty} \frac{s^{2 k}}{(2 k) !}(1-r)^{2 k}\right)\left(\sum_{k=0}^{\infty} \frac{s^{2 k}}{(2 k) !} r^{2 k} M_{2 k}\right) \\
& =p M_{\mathrm{e}}(r s)+2 q \cosh (s(1-r)) M_{\mathrm{e}}(r s)
\end{aligned}
$$

for all complex numbers $s$. A similar argument shows that

$$
M_{\mathrm{o}}(s)=p M_{\mathrm{o}}(r s)+2 q \sinh (s(1-r)) M_{\mathrm{e}}(r s)
$$

for all complex numbers $s$. Since $M(s)=M_{\mathrm{e}}(s)+M_{\mathrm{\circ}}(s)$ and $M(r s)=M_{\mathrm{e}}(r s)+M_{\mathrm{\circ}}(r s)$, it follows by adding (4.9) and (4.10) that $M(s)=p M(r s)+2 q e^{s(1-r)} M_{\mathrm{e}}(r s)$.

Below we use the following notation. If $f: \mathbb{C} \rightarrow \mathbb{C}$ is a differentiable function and $n$ is a positive integer with $n \geq 0$, then $D^{n} f$ denotes the $n$ 'th derivative of $f$.

Proposition 4.5 (Estimates of the derivatives of $\Lambda$ ). For each integer $n$ with $n \geq 0$ there is positive constant $c_{n}$ such that

$$
\left|D^{n} \Lambda(u)\right| \leq c_{n} e^{-r u}
$$

for all $u \geq 0$.

Proof We first note that if $n$ is a positive integer and $s$ is a complex number, then $D^{n} L(s)=$ $\frac{d^{n}}{d s^{n}}\left(M(s) e^{-s}\right)=\sum_{k=0}^{n}\left(\begin{array}{l}n \\ k\end{array}\right) D^{k} M(s) \frac{d^{n-k}}{d s^{n-k}}\left(e^{-s}\right)=e^{-s} \sum_{k=0}^{n}\left(\begin{array}{l}n \\ k\end{array}\right)(-1)^{n-k} D^{k} M(s)$, whence

$$
\left|D^{n} L(s)\right| \leq\left|e^{-s}\right| \sum_{k=0}^{n}\left(\begin{array}{l}
n \\
k
\end{array}\right)\left|D^{k} M(s)\right| .
$$

We now prove the following four claims.

Claim 1 For all positive integers $n$ and all $u \geq 0$, we have $\left|D^{n} M(u)\right| \leq e^{u}$.

Proof of Claim 1 For positive integers $n$ and $s \in \mathbb{C}$, we have $D^{n} M(s)=\sum_{k=0}^{\infty} \frac{M_{n+k}}{k !} s^{k}$. Since $\left|M_{k}\right| \leq 1$ for all $k$, this implies that $\left|D^{n} M(u)\right| \leq \sum_{k=0}^{\infty} \frac{\left|M_{n+k}\right|}{k !} u^{k} \leq \sum_{k=0}^{\infty} \frac{1}{k !} u^{k}=e^{u}$ for all positive integers $n$ and all $u \geq 0$. This completes the proof of Claim 1 .

For brevity write $B_{n}=\sum_{k=0}^{n}\left(\begin{array}{l}n \\ k\end{array}\right)$ for positive integers $n$ (note that $B_{n}=2^{n}$ ).

Claim 2 For all positive integers $n$ and all $u \geq 0$, we have $\left|D^{n} L(u)\right| \leq B_{n}$.

Proof of Claim 2 For positive integers $n$ and $u \geq 0$, we have using (4.11) and Claim 1, $\left|D^{n} L(u)\right| \leq e^{-u} \sum_{k=0}^{n}\left(\begin{array}{l}n \\ k\end{array}\right)\left|D^{k} M(u)\right| \leq e^{-u} \sum_{k=0}^{n}\left(\begin{array}{l}n \\ k\end{array}\right) e^{u}=B_{n}$. This completes the proof of Claim 2. 
Claim 3 For all positive integers $n$ and all $u \geq 0$, we have $\left|D^{n} M(-u)\right| \leq 1$.

Proof of Claim 3 It follows from Lemma 4.4 that if $s$ is a complex number, then $M(s)=$ $p M(r s)+2 q M_{\mathrm{e}}(r s) e^{(1-r) s}$. Differentiating this identity $n$ times gives

$$
\begin{aligned}
D^{n} M(s) & =p r^{n} D^{n} M(r s)+2 q \frac{d^{n}}{d s^{n}}\left(M_{\mathrm{e}}(r s) e^{(1-r) s}\right) \\
& =p r^{n} D^{n} M(r s)+H_{n}(s)
\end{aligned}
$$

where

$$
\begin{aligned}
H_{n}(s) & =2 q \frac{d^{n}}{d s^{n}}\left(M_{\mathrm{e}}(r s) e^{(1-r) s}\right) \\
& =2 q \sum_{i=0}^{n}\left(\begin{array}{l}
n \\
i
\end{array}\right) \frac{d^{i}}{d s^{i}}\left(M_{\mathrm{e}}(r s)\right) \frac{d^{n-i}}{d s^{n-i}}\left(e^{(1-r) s}\right) \\
& =2 q \sum_{i=0}^{n}\left(\begin{array}{l}
n \\
i
\end{array}\right) r^{i}(1-r)^{n-i} D^{i} M_{\mathrm{e}}(r s) e^{(1-r) s}
\end{aligned}
$$

Applying Lemma 4.1 therefore shows that

$$
D^{n} M(s)=\sum_{k=0}^{\infty}\left(p r^{n}\right)^{k} H_{n}\left(r^{k} s\right) .
$$

In particular, we conclude from this that if $u \geq 0$, then

$$
\begin{aligned}
\left|D^{n} M(-u)\right| & =\sum_{k=0}^{\infty}\left(p r^{n}\right)^{k}\left|H_{n}\left(-r^{k} u\right)\right| \\
& \leq 2 q \sum_{k=0}^{\infty}\left(p r^{n}\right)^{k} \sum_{i=0}^{n}\left(\begin{array}{l}
n \\
i
\end{array}\right) r^{i}(1-r)^{n-i}\left|D^{i} M_{\mathrm{e}}\left(-r^{k+1} u\right)\right| e^{-(1-r) r^{k} u}
\end{aligned}
$$

It is not difficult to see that $\left|D^{i} M_{\mathrm{e}}(s)\right| \leq e^{|s|}$ for all positive integers $i$ and all complex numbers $s$. We deduce from this and the previous inequality that if $u \geq 0$, then

$$
\begin{aligned}
\left|D^{n} M(-u)\right| & \leq 2 q \sum_{k=0}^{\infty}\left(p r^{n}\right)^{k} \sum_{i=0}^{n}\left(\begin{array}{l}
n \\
i
\end{array}\right) r^{i}(1-r)^{n-i} e^{-r^{k+1} u} e^{-(1-r) r^{k} u} \\
& =2 q \sum_{k=0}^{\infty}\left(p r^{n}\right)^{k} e^{-r^{k+1} u} e^{-(1-r) r^{k} u} \\
& =2 q \sum_{k=0}^{\infty}\left(p r^{n}\right)^{k} e^{-(1-2 r) r^{k} u}
\end{aligned}
$$

Since $r<\frac{1}{2}$, it follows that $-(1-2 r) r^{k} u<0$, whence $e^{-(1-2 r) r^{k} u} \leq 1$, and we therefore deduce from (4.12) that

$$
\left|D^{n} M(-u)\right| \leq 2 q \sum_{k \geq 0}\left(p r^{n}\right)^{k} \leq 2 q \sum_{k \geq 0} p^{k}=2 q \frac{1}{1-p}=1,
$$

where we have used the fact that $1-p=2 q$. This completes the proof of Claim 3 .

Claim 4 For all positive integers $n$ and all $u \geq 0$, we have $\left|D^{n} L(-u)\right| \leq B_{n} e^{u}$. 
Proof of Claim 4 For positive integers $n$ and $u \geq 0$, we have using (4.11) and Claim 3, $\left|D^{n} L(-u)\right| \leq e^{u} \sum_{k=0}^{n}\left(\begin{array}{l}n \\ k\end{array}\right)\left|D^{k} M(-u)\right| \leq e^{u} \sum_{k=0}^{n}\left(\begin{array}{l}n \\ k\end{array}\right)=e^{u} B_{n}$. This completes the proof of Claim 4.

We can now estimate $\left|D^{n} \Lambda(u)\right|$ for all positive integers $n$ and all $u \geq 0$. Indeed, for positive integers $n$ and $u \geq 0$, we have

$$
\begin{aligned}
D^{n} \Lambda(u)= & p \frac{d^{n}}{d u^{n}}\left(L(r u) e^{-(1-r) u}\right)+q \frac{d^{n}}{d u^{n}}\left(L(-r u) e^{-2 r u}\right) \\
= & p \sum_{k=0}^{n}\left(\begin{array}{l}
n \\
k
\end{array}\right) \frac{d^{k}}{d u^{k}}(L(r u)) \frac{d^{n-k}}{d u^{n-k}}\left(e^{-(1-r) u}\right) \\
& +q \sum_{k=0}^{n}\left(\begin{array}{l}
n \\
k
\end{array}\right) \frac{d^{k}}{d u^{k}}(L(-r u)) \frac{d^{n-k}}{d u^{n-k}}\left(e^{-2 r u}\right) \\
= & p e^{-(1-r) u} \sum_{k=0}^{n}\left(\begin{array}{l}
n \\
k
\end{array}\right) r^{k}(-(1-r))^{n-k} D^{k} L(r u) \\
& +q e^{-2 r u} \sum_{k=0}^{n}\left(\begin{array}{l}
n \\
k
\end{array}\right)(-r)^{k}(-2 r)^{n-k} D^{n} L(-r u),
\end{aligned}
$$

whence

$$
\begin{aligned}
\left|D^{n} \Lambda(u)\right| \leq & p e^{-(1-r) u} \sum_{k=0}^{n}\left(\begin{array}{l}
n \\
k
\end{array}\right) r^{k}(1-r)^{n-k}\left|D^{k} L(r u)\right| \\
& +q e^{-2 r u} \sum_{k=0}^{n}\left(\begin{array}{l}
n \\
k
\end{array}\right) r^{k}(2 r)^{n-k}\left|D^{n} L(-r u)\right| .
\end{aligned}
$$

Next, it follows from Claim 2 that if $u \geq 0$, then $\left|D^{n} L(r u)\right| \leq B_{n}$, and it follows from Claim 4 that if $u \geq 0$, then $\left|D^{n} L(-r u)\right| \leq B_{n} e^{r u}$. We conclude immediately from this and (4.13) that if $u \geq 0$, then

$$
\begin{aligned}
\left|D^{n} \Lambda(u)\right| \leq & p e^{-(1-r) u} \sum_{k=0}^{n}\left(\begin{array}{l}
n \\
k
\end{array}\right) r^{k}(1-r)^{n-k} B_{n} \\
& +q e^{-2 r u} \sum_{k=0}^{n}\left(\begin{array}{l}
n \\
k
\end{array}\right) r^{k}(2 r)^{n-k} B_{n} e^{r u} .
\end{aligned}
$$

Finally, Since $\sum_{K=0}^{N}\left(\begin{array}{l}N \\ K\end{array}\right) R^{K}(1-R)^{N-K}=1$ And $\sum_{K=0}^{N}\left(\begin{array}{l}N \\ K\end{array}\right)(R)^{K}(2 R)^{N-K}=(3 R)^{N}$, it follows from (4.14) that

$$
\left|D^{n} \Lambda(u)\right| \leq p B_{n} e^{-(1-r) u}+q B_{n}(3 r)^{n} e^{-r u} .
$$

The desired result follows immediately from the above inequality since $r<1-r$.

Proposition 4.6 (Estimates of the integral of $\Lambda$ ). There is a constant $c$ such that

$$
\int_{0}^{\infty}\left|\Lambda\left(r^{k} u\right) u^{s-1}\right| d u \leq c \Gamma(\operatorname{Re} s) \frac{1}{\rho^{\operatorname{Re} s} r^{k+1 \operatorname{Re} s}}
$$

for all integers $k$ with $k \geq 0$ and all $s \in \mathbb{C}$ with $\operatorname{Re} s>0$ (here $\Gamma(\operatorname{Re} s)$ denotes the Gamma-function evaluated at $\operatorname{Re} s$ ). 
Proof Fix $s \in \mathbb{C}$ with $\operatorname{Re} s>0$. We conclude from Proposition 4.5 that there is a constant $c$ such that $|\Lambda(u)| \leq c e^{-r u}$ for all $u \geq 0$. This implies that

$$
\begin{aligned}
\int_{0}^{\infty}\left|\Lambda\left(r^{k} u\right) u^{s-1}\right| d u & =\int_{0}^{\infty}\left|\Lambda\left(r^{k} u\right)\right| u^{\operatorname{Re} s-1} d u \\
& \leq c \int_{0}^{\infty} e^{-r^{k+1} u} u^{\operatorname{Re} s-1} d u .
\end{aligned}
$$

Next, introducing the substitution $v=\rho r^{k} u$ into the integral $\int_{0}^{\infty} e^{-r^{k+1} u} u^{\operatorname{Re} s-1} d u$ in (4.15) shows that

$$
\begin{aligned}
\int_{0}^{\infty}\left|\Lambda\left(r^{k} u\right) u^{s-1}\right| d u & \leq c \frac{1}{\left(r^{k+1}\right)^{\operatorname{Re} s}} \int_{0}^{\infty} e^{-v} v^{\operatorname{Re} s-1} d v \\
& =c \Gamma(\operatorname{Re} s) \frac{1}{\left(r^{k+1}\right)^{\operatorname{Re} s}} .
\end{aligned}
$$

This completes the proof.

\section{Proof of Theorems 2.4 and 2.5: the proof of (2.7)}

The purpose of this section is to prove (2.7), namely, that there is a constant $K$ such that

$$
\left|M_{n}-L(n)\right| \leq K \frac{1}{n^{\Delta+1}}
$$

for all positive integers $n$ (recall, that $\Delta=\frac{\log q}{\log r}$ where $q=p_{1} p_{2}$ ). The key tool for proving this inequality is Theorem 5.1 below. For $s \in \mathbb{C}$ with $s \neq 0$, let $\arg s$ denote the unique argument of $s$ with $\arg s \in[-\pi, \pi)$, and for $\theta \in[-\pi, \pi)$, write

$$
\Xi_{\Theta}=\{s \in \mathbb{C} \backslash\{0\}|| \arg s \mid \leq \theta\} .
$$

Theorem 5.1 [15, p. 14, Theorem 1] Let $\left(t_{n}\right)_{n}$ be a sequence of bounded positive numbers and define $f: \mathbb{C} \rightarrow \mathbb{C}$ by

$$
f(S)=\sum_{n=0}^{\infty} \frac{t_{n}}{n !} s^{n} e^{-s} .
$$

Assume that there are positive constants $R_{0}, R_{1}, A_{0}, A_{1}, D, \theta$ and $\delta$ with $\theta<\frac{\pi}{2}$ and $\delta<1$ such that the following hold:

(1) If $s \in \Xi_{\theta}$ and $|s|>R_{0}$, then $|f(s)| \leq A_{0} \frac{1}{|s|^{D}}$;

(2) If $s \notin \Xi_{\theta}$ and $|s|>R_{1}$, then $\left|f(s) e^{s}\right| \leq A_{1} e^{\delta|s|}$.

Then there is a constant $K$ such that

$$
\left|t_{n}-f(n)\right| \leq K \frac{1}{n^{D+1}}
$$

for all $n$.

Theorem 5.2 Recall that the function $L: \mathbb{C} \rightarrow \mathbb{C}$ is defined by

$$
L(s)=\sum_{n=0}^{\infty} \frac{M_{n}}{n !} s^{n} e^{-s} .
$$


There are positive constants $R_{0}, R_{1}, A_{0}, A_{1}$ and $\delta$ with $\delta<1$ such that the following hold:

(1) If $s \in \Xi_{\frac{\pi}{4}}$ and $|s|>R_{0}$, then $|L(s)| \leq A_{0} \frac{1}{|s|^{\Delta}}$;

(2) If $s \notin \Xi_{\frac{\pi}{4}}$ and $|s|>R_{1}$, then $\left|L(s) e^{s}\right| \leq A_{1} e^{\delta|s|}$.

Proof (1) We first prove the following three claims.

Claim 1 For all $s \in \mathbb{C}$, the series $\sum_{k=0}^{\infty} p^{k} L_{\mathrm{e}}\left(r^{k+1} s\right) \frac{1}{e^{\left(1-r^{k}\right) s}}$ converges and $L(s)=$ $2 q \sum_{k=0}^{\infty} p^{k} L_{\mathrm{e}}\left(r^{k+1} s\right) \frac{1}{e^{\left(1-r^{k}\right) s}}$.

Proof of Claim 1 It follows from Lemma 4.4 and the definition of $L_{\mathrm{e}}$ that $M(s)=p M(r s)+$ $2 q M_{\mathrm{e}}(r s) e^{(1-r) s}=p M(r s)+2 q L_{\mathrm{e}}(r s) e^{s}=p M(r s)+P(s)$ for all $s \in \mathbb{C}$ where $P(s)=$ $2 q L_{\mathrm{e}}(r s) e^{s}$. This and Lemma 4.1 now implies that $L(s) e^{s}=M(s)=\sum_{p=0}^{\infty} p^{k} P\left(r^{k} s\right)=$ $2 q \sum_{k=0}^{\infty} p^{k} L_{\mathrm{e}}\left(r^{k+1} s\right) e^{r^{k} s}$. This completes the proof of Claim 1.

Define $Q: \mathbb{C} \rightarrow \mathbb{C}$ by

$$
Q(s)=\left(p e^{-(1-r) s}+q e^{-2(1-r) s}\right) L_{e}(r s) .
$$

Claim 2 For all $s \in \mathbb{C}$, the series $\sum_{l=0}^{\infty} q^{l} Q\left(r^{l} s\right)$ converges and $L_{e}(s)=\sum_{l=0}^{\infty} q^{l} Q\left(r^{l} s\right)$.

Proof of Claim 2 Lemma 4.4 shows that $M_{\mathrm{e}}(s)=p M_{\mathrm{e}}(r s)+2 q \cosh ((1-r) s) M_{\mathrm{e}}(r s)$ for all $s \in \mathbb{C}$. It follows from this and a lengthy, but straight forward, calculation that

$$
\begin{aligned}
L_{\mathrm{e}}(s) & =M_{\mathrm{e}}(s) e^{-s} \\
& =\left(p M_{\mathrm{e}}(r s)+2 q \cosh ((1-r) s) M_{\mathrm{e}}(r s)\right) e^{-s} \\
& =q L_{\mathrm{e}}(r s)+\left(p e^{-(1-r) s}+q e^{-2(1-r) s}\right) L_{\mathrm{e}}(r s) \\
& =q L_{\mathrm{e}}(r s)+Q(s)
\end{aligned}
$$

for all $s \in \mathbb{C}$. This and Lemma 4.1 now implies that $L_{\mathrm{e}}(s)=\sum_{l=0}^{\infty} q^{l} Q\left(r^{l} s\right)$. This completes the proof of Claim 2.

Claim 3 For all $s \in \Xi_{\frac{\pi}{4}}$, we have $|Q(s)| \leq(p+q) e^{-\frac{1}{8}|s|}$.

Proof of Claim 3 For $s \in \mathbb{C}$, we have

$$
\begin{aligned}
|Q(s)| & =\left|\left(p e^{-(1-r) s}+q e^{-2(1-r) s}\right) L_{\mathrm{e}}(r s)\right| \\
& \leq\left(p\left|e^{-(1-r) s}\right|+q\left|e^{-2(1-r) s}\right|\right)\left|L_{\mathrm{e}}(r s)\right| \\
& \leq\left(p e^{-(1-r) \operatorname{Re} s}+q e^{-2(1-r) \operatorname{Re} s}\right)\left|L_{\mathrm{e}}(r s)\right| .
\end{aligned}
$$

Next, observe that $\left|L_{\mathrm{e}}(r s)\right|=\left|M_{\mathrm{e}}(r s)\right|\left|e^{-r s}\right|=\left|\sum_{k=0}^{\infty} \frac{(r s)^{2 k}}{(2 k) !} M_{2 k}\right| e^{-r \operatorname{Re} s} \leq\left(\sum_{k=0}^{\infty} \frac{(r|s|)^{2 k}}{(2 k) !}\right)$ $e^{-r \operatorname{Re} s} \leq\left(\sum_{k=0}^{\infty} \frac{(r|s|)^{k}}{k !}\right) e^{-r \operatorname{Re} s}=e^{r|s|} e^{-r \operatorname{Re} s}=e^{r|s|-r \operatorname{Re} s}$ since $M_{k} \leq 1$ for all $k$. This and (5.1) imply that if $s \in \mathbb{C}$, then

$$
\begin{aligned}
|Q(s)| & \leq\left(p e^{-(1-r) \operatorname{Re} s}+q e^{-2(1-r) \operatorname{Re} s}\right)\left|L_{\mathrm{e}}(r s)\right| \\
& \leq\left(p e^{-(1-r) \operatorname{Re} s}+q e^{-2(1-r) \operatorname{Re} s}\right) e^{r|s|-r \operatorname{Re} s} .
\end{aligned}
$$


Also, if $s \in \Xi_{\frac{\pi}{4}}$, then $\operatorname{Re} s \geq 0$, whence $-2(1-r) \operatorname{Re} s \leq-(1-r) \operatorname{Re} s$, and so $e^{-2(1-r) \operatorname{Re} s} \leq e^{-(1-r) \operatorname{Re} s}$. We deduce from this and (5.2) that if $s \in \Xi_{\frac{\pi}{4}}$, then

$$
|Q(s)| \leq\left(p e^{-(1-r) \operatorname{Re} s}+q e^{-(1-r) \operatorname{Re} s}\right) e^{r|s|-r \operatorname{Re} s}=(p+q) e^{r|s|-\operatorname{Re} s} .
$$

We now note that if $s \in \Xi_{\frac{\pi}{4}}$, then $\operatorname{Re} s=|s| \cos \arg s$ where $|\arg s| \leq \frac{\pi}{4}$, whence (since $\left.r \leq \frac{1}{2}\right) r-\cos \arg s \leq \frac{1}{2}-\cos \frac{\pi}{4}=\frac{1}{2}-\frac{\sqrt{2}}{2} \leq-\frac{1}{8}$, and so $r|s|-\operatorname{Re} s=r|s|-|s| \cos \arg s=$ $|s|(r-\cos \arg s) \leq-\frac{1}{8}|s|$. It finally follows from this and (5.3) that if $s \in \Xi_{\frac{\pi}{4}}$, then

$$
|Q(s)| \leq(p+q) e^{r|s|-\operatorname{Re} s} \leq(p+q) e^{-\frac{1}{8}|s|} .
$$

This completes the proof of Claim 3.

Combining Claims 1 and 2 we deduce that if $s \in \mathbb{C}$, then

$$
\begin{aligned}
|L(s)| & =\left|2 q \sum_{k=0}^{\infty} p^{k} L_{\mathrm{e}}\left(r^{k+1} s\right) \frac{1}{e^{\left(1-r^{k}\right) s}}\right| \\
& \leq 2 q \sum_{k=0}^{\infty} p^{k}\left|L_{\mathrm{e}}\left(r^{k+1} s\right)\right| \frac{1}{e^{\left(1-r^{k}\right) \operatorname{Re} s}} \\
& =2 q \sum_{k=0}^{\infty} p^{k}\left|\sum_{l=0}^{\infty} q^{l} Q\left(r^{l+k+1} s\right)\right| \frac{1}{e^{\left(1-r^{k}\right) \operatorname{Re} s}} \\
& \leq 2 q \sum_{k=0}^{\infty} \sum_{l=0}^{\infty} p^{k} q^{l}\left|Q\left(r^{l+k+1} s\right)\right| \frac{1}{e^{\left(1-r^{k}\right) \operatorname{Re} s}} .
\end{aligned}
$$

Next, we observe that if $s \in \Xi_{\frac{\pi}{4}}$, then $r^{m} s \in \Xi_{\frac{\pi}{4}}$ for all integers $m$. Using Claim 3 we therefore deduce from (5.4) that if $s \in \Xi_{\frac{\pi}{4}}$, then

$$
\begin{aligned}
|L(s)| & \leq 2 q \sum_{k=0}^{\infty} \sum_{l=0}^{\infty} p^{k} q^{l}\left|Q\left(r^{l+k+1} s\right)\right| \frac{1}{e^{\left(1-r^{k}\right) \operatorname{Re} s}} \\
& \leq 2 q(p+q) \sum_{k=0}^{\infty} \sum_{l=0}^{\infty} p^{k} q^{l} e^{-\frac{1}{8} r^{l+k+1}|s|} \frac{1}{e^{\left(1-r^{k}\right) \operatorname{Re} s}} \\
& =2 q(p+q)\left(\sum_{l=0}^{\infty} q^{l} e^{-\frac{1}{8} r^{l+1}|s|}+\sum_{k=1}^{\infty} \sum_{l=0}^{\infty} p^{k} q^{l} e^{-\frac{1}{8} r^{l+k+1}|s|} \frac{1}{e^{\left(1-r^{k}\right) \operatorname{Re} s}}\right) \\
& =2 q(p+q)\left(U_{0}(s)+U_{1}(s)\right),
\end{aligned}
$$

where

$$
\begin{aligned}
& U_{0}(s)=\sum_{l=0}^{\infty} q^{l} e^{-\frac{1}{8} r^{l+1}|s|}, \\
& U_{1}(s)=\sum_{k=1}^{\infty} \sum_{l=0}^{\infty} p^{k} q^{l} e^{-\frac{1}{8} r^{l+k+1}|s|} \frac{1}{e^{\left(1-r^{k}\right) \operatorname{Re} s}} .
\end{aligned}
$$

We will now estimate $U_{0}(s)$ and $U_{1}(s)$; this is done in Claims 4 and 5 below.

Claim 4 There is a constant $k_{0}$ such that $U_{0}(s) \leq k_{0} \frac{1}{|s|^{\Delta}}$ for all $s \in \mathbb{C}$. 
Proof of Claim 4 It is easily seen that

$$
U_{0}(s)=\sum_{l=0}^{\infty} q^{l} e^{-\frac{1}{8} r^{l+1}|s|} \leq 2 \int_{0}^{\infty} q^{x} e^{-\frac{1}{8} r^{x+1}|s|} d x .
$$

Introducing the substitution $y=-\frac{1}{8} r^{x+1}|s|$ into the integral $\int_{0}^{\infty} q^{x} e^{-\frac{1}{8} r^{x+1}|s|} d x$ in (5.6) yields

$$
\begin{aligned}
U_{0}(s) & \leq 2 \int_{0}^{\infty} q^{x} e^{-\frac{1}{8} r^{x+1}|s|} d x \\
& =2 \frac{1}{-\log r}\left(\frac{r}{8}\right)^{-\Delta} \int_{0}^{\frac{1}{8} r|s|} y^{\Delta-1} e^{-y} d y \frac{1}{|s|^{\Delta}} \\
& \leq 2 \frac{1}{-\log r}\left(\frac{r}{8}\right)^{-\Delta} \int_{0}^{\infty} y^{\Delta-1} e^{-y} d y \frac{1}{|s|^{\Delta}} \\
& =2 \frac{1}{-\log r}\left(\frac{r}{8}\right)^{-\Delta} \Gamma(\Delta) \frac{1}{|s|^{\Delta}} \\
& =k_{0} \frac{1}{|s|^{\Delta}}
\end{aligned}
$$

for $s \in \mathbb{C}$ where $k_{0}=2 \frac{1}{-\log r}\left(\frac{r}{8}\right)^{-\Delta} \Gamma(\Delta)$ (here $\Gamma(\Delta)=\int_{0}^{\infty} y^{\Delta-1} e^{-y} d y$ denotes the Gamma-function evaluated at $\Delta$ ). This completes the proof of Claim 4.

Claim 5 There is a constant $k_{1}$ such that $U_{1}(s) \leq k_{1} \frac{1}{e^{\frac{\sqrt{2}}{2}(1-r)|s|}}$ for all $s \in \Xi_{\frac{\pi}{4}}$.

Proof of Claim 5 Fix $s \in \Xi_{\frac{\pi}{4}}$. Since Re $s \geq 0$ (because $s \in \Xi_{\frac{\pi}{4}}$ ), we conclude that (1 $\left.r^{k}\right) \operatorname{Re} s \geq(1-r) \operatorname{Re} s$, whence $\frac{1}{e^{\left(1-r^{k}\right) \operatorname{Re} s}} \leq \frac{1}{e^{(1-r) \operatorname{Re} s}}$ for all integers $k$ with $k \geq 1$. We deduce from this and the fact that $e^{-\frac{1}{8} r^{l+k+1}|s|} \leq 1$ for all integers $k$ and $l$ that

$$
\begin{aligned}
U_{1}(s) & =\sum_{k=1}^{\infty} \sum_{l=0}^{\infty} p^{k} q^{l} e^{-\frac{1}{8} r^{l+k+1}|s|} \frac{1}{e^{\left(1-r^{k}\right) \operatorname{Re} s}} \\
& \leq \sum_{k=1}^{\infty} \sum_{l=0}^{\infty} p^{k} q^{l} \frac{1}{e^{(1-r) \operatorname{Re} s}} \\
& =k_{1} \frac{1}{e^{(1-r) \operatorname{Re} s}}
\end{aligned}
$$

where $k_{1}=\sum_{k=1}^{\infty} \sum_{l=0}^{\infty} p^{k} q^{l}=\left(\sum_{k=1}^{\infty} p^{k}\right)\left(\sum_{l=0}^{\infty} q^{l}\right)<\infty$. Also, since $s \in \Xi_{\frac{\pi}{4}}$, it follows that $\operatorname{Re} s=|s| \cos \arg s \geq|s| \cos \frac{\pi}{4}=\frac{\sqrt{2}}{2}|s|$, and so $\frac{1}{e^{(1-r) \operatorname{Re} s}} \leq \frac{1}{e^{\frac{\sqrt{2}}{2}(1-r)|s|}}$. We deduce from this and (5.7) that

$$
U_{1}(s) \leq k_{1} \frac{1}{e^{(1-r) \operatorname{Re} s}} \leq k_{1} \frac{1}{e^{\frac{\sqrt{2}}{2}(1-r)|s|}} .
$$

This completes the proof of Claim 5.

Finally, it follows immediately from (5.5), Claims 4 and 5 that if $s \in \Xi_{\frac{\pi}{4}}$, then

$$
|L(s)| \leq 2 q(p+q)\left(U_{0}(s)+U_{1}(s)\right) \leq 2 q(p+q)\left(k_{0} \frac{1}{|s|^{\Delta}}+k_{1} \frac{1}{e^{\frac{\sqrt{2}}{2}(1-r)|s|}}\right)
$$

for all complex numbers $s$. The desired result follows easily from this. 
(2) Lemma 4.4 shows that $L(s) e^{s}=M(s)=p M(s)+2 q M_{\mathrm{e}}(r s) e^{(1-r) s}$ for $s \in \mathbb{C}$, whence

$$
\left|L(s) e^{s}\right| \leq p|M(r s)|+2 q\left|M_{\mathrm{e}}(r s)\right|\left|e^{(1-r) s}\right|=p|M(r s)|+2 q\left|M_{\mathrm{e}}(r s)\right| e^{(1-r) \operatorname{Re} s}(5.8)
$$

for $s \in \mathbb{C}$. Since $|M(z)|=\left|\sum_{k=0}^{\infty} \frac{z^{k}}{k !} M_{k}\right| \leq \sum_{k=0}^{\infty} \frac{|z|^{k}}{k !}=e^{|z|}$ and $\left|M_{\mathrm{e}}(z)\right|=$ $\left|\sum_{k=0}^{\infty} \frac{z^{2 k}}{(2 k) !} M_{2 k}\right| \leq \sum_{k=0}^{\infty} \frac{|z|^{2 k}}{(2 k) !} \leq \sum_{k=0}^{\infty} \frac{|z|^{k}}{k !}=e^{|z|}$ for $z \in \mathbb{C}$, we now conclude from (5.8) that

$$
\left|L(s) e^{s}\right| \leq p e^{r|s|}+2 q e^{r|s|+(1-r) \operatorname{Re} s}
$$

for $s \in \mathbb{C}$. However, if $s \notin \Xi_{\frac{\pi}{4}}$ and $s \neq 0$, then $|\arg s|>\frac{\pi}{4}$, whence $\cos \arg s \leq \frac{\sqrt{2}}{2}$, and so $r|s|+(1-r) \operatorname{Re} s=r|s|+(1-r)|s| \cos \arg s \leq r|s|+(1-r)|s| \frac{\sqrt{2}}{2}=\frac{(2-\sqrt{2}) r+\sqrt{2}}{2}|s| \leq$ $\frac{(2-\sqrt{2}) \frac{1}{2}+\sqrt{2}}{2}|s|=\frac{2+\sqrt{2}}{4}|s| \leq \frac{7}{8}|s|$. Since also $r|s| \leq \frac{1}{2}|s| \leq \frac{7}{8}|s|$ for all $s \in \mathbb{C}$, we therefore conclude from (5.9) that if $s \notin \Xi_{\frac{\pi}{4}}$ and $s \neq 0$, then

$$
\left|L(s) e^{s}\right| \leq p e^{\frac{7}{8}|s|}+2 q e^{\frac{7}{8}|s|}=(p+2 q) e^{\frac{7}{8}|s|} .
$$

This completes the proof.

Theorem 5.3 There is a constant $K$ such that

$$
\left|M_{n}-L(n)\right| \leq K \frac{1}{n^{\Delta+1}}
$$

for all $n$.

Proof This follows immediately from Theorems 5.1 and 5.2.

\section{Proof of Theorems 2.4 and 2.5: proof of (2.8)}

The purpose of this section is to prove inequality (2.8), namely, that for each real number $d$ with $d>\Delta$ (recall that $\Delta=\frac{\log q}{\log r}=\frac{\log p_{1} p_{2}}{\log r}$ ) there is a constant $K_{d}$ such that

$$
\left|L(u)-u^{-\Delta} \Pi(u)\right| \leq K_{d} \frac{1}{u^{d}}
$$

for all $u>0$ where $\Pi$ is the function defined in Theorem 2.4 (or, alternatively, in Theorem 6.5 below). The proof of (6.1) is divided in the following four parts:

Part 1 We first define the moment zeta-function

$$
Z:\{s \in \mathbb{C} \mid \operatorname{Re} s>0\} \rightarrow \mathbb{C}:
$$

this is done in Theorem and Definition 6.1.

Part 2 Next, using the Mellin transform theory, we show that $L$ can be written as a complex curve integral involving $Z$, namely, we show that for $0<c<\Delta$ we have

$$
L(u)=\frac{1}{2 \pi \dot{\mathrm{i}}} \int_{c-\dot{\mathrm{i}} \infty}^{c+\mathrm{i} \infty} \frac{Z(s)}{1-q r^{-s}} u^{-s} d s
$$

for all $u>0$ (recall that $q=p_{1} p_{2}$ ); this is done in Theorem 6.3. 
Part 3 Finally, using the residue theorem, we compute the complex curve integral in (6.2). In particular, we show that if $0<c<\Delta<d$, then

$$
\frac{1}{2 \pi \dot{\mathrm{i}}} \int_{c-\dot{\mathrm{i}} \infty}^{c+\mathrm{i} \infty} \frac{Z(s)}{1-q r^{-s}} u^{-s} d s=u^{-\Delta} \Pi(u)+\mathcal{O}\left(\frac{1}{u^{d}}\right)
$$

for all $u>0$; this is done in Theorems 6.4 and 6.6: in Theorems 6.4 and 6.5 estimates for $|Z(s)|$ and $\left|1-q r^{-s}\right|$ are obtained and in Theorem 6.6 we use the residue theorem together with the estimates from Theorems 6.4 and 6.5 to derive formula (6.3).

Part 4 The desired inequality [i.e. (6.1)] follows immediately from combining (6.2) and (6.3).

We now define the moment zeta-function $Z$.

Theorem and Definition 6.1 (The moment zeta-function) For $s \in \mathbb{C}$ with $0<\operatorname{Re} s$, we have

$$
\int_{0}^{\infty}\left|\Lambda(u) u^{s-1}\right| d u<\infty
$$

In particular, the moment zeta function $Z:\{s \in \mathbb{C} \mid \operatorname{Re} s>0\} \rightarrow \mathbb{C}$ defined by

$$
Z(s)=\int_{0}^{\infty} \Lambda(u) u^{s-1} d u
$$

is well-defined.

Proof This follows from Proposition 4.6.

Next, using the Mellin transform theory, we show that the function $L$ can be expressed as a complex curve integral involving the moment zeta-function $Z$. For the benefit of the reader we first state the Mellin transform theorem.

Theorem 6.2 (The Mellin transform theorem [19]) Let $a, b \in[-\infty, \infty]$ with $a<b$ and let $f:(0, \infty) \rightarrow \mathbb{R}$ be a real valued function. Assume that the following conditions are satisfied:

(i) The function $f$ is piecewise continuous on all compact subintervals of $(0, \infty)$, and at

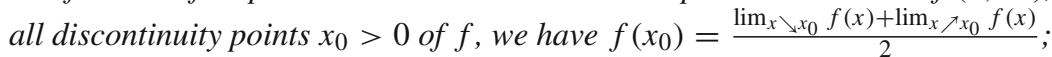

(ii) If $s \in \mathbb{C}$ satisfies $a<\operatorname{Re} s<b$, then $\int_{0}^{\infty}\left|x^{s-1} f(x)\right| d x<\infty$.

Then we have:

(1) Fors $\in \mathbb{C}$ with $a<\operatorname{Re} s<b$ the integral

$$
\int_{0}^{\infty} x^{s-1} f(x) d x
$$

is well-defined.

It follows from (1) that the function $\mathrm{M} f:\{s \in \mathbb{C} \mid a<\operatorname{Re} s<b\} \rightarrow \mathbb{C}$ given by

$$
(\mathrm{M} f)(s)=\int_{0}^{\infty} x^{s-1} f(x) d x
$$

is well-defined. The function $\mathrm{M} f$ is called the Mellin transform of $f$. 
(2) For $c \in \mathbb{R}$ with $a<c<b$ and $x>0$ the integral

$$
\int_{c-i \dot{\infty} \infty}^{c+\mathrm{i} \infty} x^{-s}(\mathrm{M} f)(s) d s
$$

is well-defined.

(3) For $c \in \mathbb{R}$ with $a<c<b$ and $x>0$, we have

$$
f(x)=\frac{1}{2 \pi \dot{\mathrm{i}}} \int_{c-\dot{\mathrm{i}} \infty}^{c+\dot{\mathrm{i}} \infty} x^{-s}(\mathrm{M} f)(s) d s .
$$

Theorem 6.3 (1) For $s \in \mathbb{C}$ with $0<\operatorname{Re} s<\Delta$, we have $\int_{0}^{\infty}\left|L(u) u^{s-1}\right| d u<\infty$. In particular, the Mellin transform $\mathrm{ML}:\{s \in \mathbb{C} \mid 0<\operatorname{Re} s<\Delta\} \rightarrow \mathbb{C}$ of L defined by

$$
(\mathrm{M} L)(s)=\int_{0}^{\infty} L(u) u^{s-1} d u
$$

is well-defined.

(2) For $s \in \mathbb{C}$ with $0<\operatorname{Re} s<\Delta$, we have

$$
(\mathrm{M} L)(s)=\frac{Z(s)}{1-q r^{-s}} .
$$

(3) For $c \in \mathbb{R}$ with $0<c<\Delta$ and $u>0$ the integral $\int_{c-i \infty}^{c+i \infty} u^{-s}(\mathrm{ML})(s) d s$ is well-defined and we have

$$
L(u)=\frac{1}{2 \pi \dot{\mathrm{i}}} \int_{c-\dot{\mathrm{i} \infty}}^{c+\mathrm{i} \infty} u^{-s}(\mathrm{M} L)(s) d s .
$$

In particular, for $c \in \mathbb{R}$ with $0<c<\Delta$ and $u>0$, we have

$$
L(u)=\frac{1}{2 \pi \dot{\mathrm{i}}} \int_{c-\dot{\mathrm{i} \infty}}^{c+\dot{\mathrm{i}} \infty} \frac{Z(s)}{1-q r^{-s}} u^{-s} d s .
$$

Proof (1)-(2) We first note that a simple calculation shows that $L(s)=q L(r s)+\Lambda(s)$ for $s \in \mathbb{C}$, and it therefore follows from Lemma 4.1 that the series $\sum_{k=0}^{\infty} q^{k} \Lambda\left(r^{k} s\right)$ converges and that

$$
L(s)=\sum_{k=0}^{\infty} q^{k} \Lambda\left(r^{k} s\right)
$$

for all $s \in \mathbb{C}$.

Now fix $s \in \mathbb{C}$ with $0<\operatorname{Re} s<\Delta$, and define the functions $f_{n}, f, g:(0, \infty) \rightarrow \mathbb{C}$ for positive integers $n$ by

$$
\begin{aligned}
f_{n}(u) & =\sum_{k=0}^{n} q^{k} \Lambda\left(r^{k} s\right) u^{s-1}, \\
f(u) & =L(u) u^{s-1}=\sum_{k=0}^{\infty} q^{k} \Lambda\left(r^{k} s\right) u^{s-1}, \\
g(u) & =\sum_{k=0}^{\infty} q^{k}\left|\Lambda\left(r^{k} s\right) u^{s-1}\right|
\end{aligned}
$$

Since $\operatorname{Re} s<\Delta=\frac{\log q}{\log r}$, we conclude that $\frac{q}{r^{\operatorname{Re} s}}<1$, whence $\sum_{k=0}^{\infty}\left(\frac{q}{r^{\operatorname{Re} s}}\right)^{k}<\infty$. This and Proposition 4.6 imply that 


$$
\begin{aligned}
\int_{0}^{\infty} g(u) d u & =\int_{0}^{\infty} \sum_{k=0}^{n} q^{k}\left|\Lambda\left(r^{k} s\right) u^{s-1}\right| d u \\
& \leq \sum_{k=0}^{\infty} q^{k} \int_{0}^{\infty}\left|\Lambda\left(r^{k} u\right)\right| u^{\operatorname{Re} s-1} d u \\
& \leq c \Gamma(\operatorname{Re} s) \frac{1}{r^{\operatorname{Re} s}} \sum_{k=0}^{\infty}\left(\frac{q}{r^{\operatorname{Re} s}}\right)^{k} \\
& <\infty .
\end{aligned}
$$

We also note that $f_{n}(u) \rightarrow f(u)$ for all $u \in(0, \infty)$ and that $\left|f_{n}\right| \leq g$ for all $n$. Since $\iint_{0}^{\infty} g(u) d u<\infty$ [by (6.5)], we now conclude from this and the dominated convergence theorem that $\int_{0}^{\infty}\left|L(u) u^{s-1}\right| d u=\int_{0}^{\infty}|f(u)| d u<\infty$ and that $\int_{0}^{\infty} f(u) d u=$ $\int_{0}^{\infty} \lim _{n} f_{n}(u) d u=\lim _{n} \int_{0}^{\infty} f_{n}(u) d u$, whence

$$
\begin{aligned}
(\mathrm{ML})(s) & =\int_{0}^{\infty} L(u) u^{s-1} d u \\
& =\int_{0}^{\infty} f(u) d u \\
& =\lim _{n} \int_{0}^{\infty} f_{n}(u) d u \\
& =\lim _{n} \int_{0}^{\infty} \sum_{k=0}^{n} q^{k} \Lambda\left(r^{k} u\right) u^{s-1} d u \\
& =\lim _{n} \sum_{k=0}^{n} q^{k} \int_{0}^{\infty} \Lambda\left(r^{k} u\right) u^{s-1} d u \\
& =\lim _{n} \sum_{k=0}^{n}\left(\frac{q}{r^{s}}\right)^{k} \int_{0}^{\infty} \Lambda(v) v^{s-1} d v \\
& =\sum_{k=0}^{\infty}\left(\frac{q}{r^{s}}\right)^{k} Z(s) \\
& =\frac{1}{1-q r^{-s}} Z(s) .
\end{aligned}
$$

(3) This statement follows from Theorem 6.2.

Finally, using the residue theorem, we compute the complex curve integral in (6.4). This is done in Theorems 6.4, 6.5 and 6.6: in Theorems 6.4 and 6.5 estimates for $|Z(s)|$ and $\left|1-q r^{-s}\right|$ are obtained and in Theorem 6.6 we use the residue theorem together with the estimates from Theorems 6.4 and 6.5 to compute the curve integral in (6.4).

Theorem 6.4 For a real number $d$ with $0<d$, write

$$
\begin{aligned}
\mathbb{H}_{d}= & \{s \in \mathbb{C} \mid 0<\operatorname{Re} s \leq d\}, \\
\mathbb{K}_{d}= & \left\{s \in \mathbb{C} \mid 0<\operatorname{Re} s \leq d, \operatorname{Im} s \in \frac{2 \pi\left(\mathbb{N}-\frac{1}{2}\right)}{\log r} \cup \frac{2 \pi\left(-\left(\mathbb{N}-\frac{1}{2}\right)\right)}{\log r}\right\} \\
& \cup\{s \in \mathbb{C} \mid \operatorname{Re} s=d\} .
\end{aligned}
$$


(1) For all real numbers $d$ with $0<d$, there is a constant $h_{d}$ such that

$$
|Z(s)| \leq h_{d} \frac{1}{|s|^{2}}
$$

for all $s \in \mathbb{H}_{d}$.

(2) For all real numbers $d$ with $0<d$ and $d \neq \Delta$, there is a constant $k_{d}$ such that

$$
\frac{1}{\left|1-q r^{-s}\right|} \leq k_{d}
$$

for all $s \in \mathbb{K}_{d}$.

Proof (1) For $s \in \mathbb{C}$ with $\operatorname{Re} s>0$, we have

$$
\begin{aligned}
Z(s)= & \int_{0}^{\infty} \Lambda(u) u^{s-1} d u \\
= & \lim _{n} \int_{\frac{1}{n}}^{n} \Lambda(u) u^{s-1} d u \\
= & \lim _{n}\left(\frac{1}{s}\left[\Lambda(u) u^{s}\right]_{u=\frac{1}{n}}^{u=n}-\frac{1}{s} \int_{\frac{1}{n}}^{n} \Lambda^{\prime}(u) u^{s} d u\right) \\
= & \lim _{n}\left(\frac{1}{s}\left[\Lambda(u) u^{s}\right]_{u=\frac{1}{n}}^{u=n}-\frac{1}{s(s+1)}\left[\Lambda^{\prime}(u) u^{s+1}\right]_{u=\frac{1}{n}}^{u=n}\right. \\
& \left.+\frac{1}{s(s+1)} \int_{\frac{1}{n}}^{n} \Lambda^{\prime \prime}(u) u^{s+1} d u\right)
\end{aligned}
$$

However, it follows from Proposition 4.5 that for each integer $n$, there is a positive constant $c_{n}$ such that $\left|D^{n} \Lambda(u)\right| \leq c_{n} e^{-r u}$ for all $u \geq 0$. In particular, this implies that there are constants $c_{0}$ and $c_{1}$ such that if $s \in \mathbb{C}$, with $\operatorname{Re} s>0$, then

$$
\left|\Lambda(u) u^{s}\right| \leq c_{0} e^{-r u} u^{\operatorname{Re} s} \rightarrow 0 \text { as } u \rightarrow 0 \text { and as } u \rightarrow \infty,
$$

and

$$
\left|\Lambda^{\prime}(u) u^{s+1}\right| \leq c_{1} e^{-r u} u^{\operatorname{Re} s+1} \rightarrow 0 \text { as } u \rightarrow 0 \text { and as } u \rightarrow \infty .
$$

We conclude immediately from this that

$$
\left[\Lambda(u) u^{s}\right]_{u=\frac{1}{n}}^{u=n} \rightarrow 0 \text { as } n \rightarrow \infty \text { and }\left[\Lambda^{\prime}(u) u^{s+1}\right]_{u=\frac{1}{n}}^{u=n} \rightarrow 0 \text { as } n \rightarrow \infty
$$

and (6.7) therefore shows that

$$
Z(s)=\lim _{n} \frac{1}{s(s+1)} \int_{\frac{1}{n}}^{n} \Lambda^{\prime \prime}(u) u^{s+1} d u .
$$

Hence, for all $s \in \mathbb{C}$ with $\operatorname{Re} s>0$, we have

$$
\begin{aligned}
|Z(s)| & \leq \limsup _{n}\left|\frac{1}{s(s+1)} \int_{\frac{1}{n}}^{n} \Lambda^{\prime \prime}(u) u^{s+1} d u\right| \\
& \leq \limsup _{n} \frac{1}{|s(s+1)|} \int_{\frac{1}{n}}^{n}\left|\Lambda^{\prime \prime}(u) u^{s+1}\right| d u \mid \\
& =\frac{1}{|s|^{2}} \int_{0}^{\infty}\left|\Lambda^{\prime \prime}(u) u^{s+1}\right| d u .
\end{aligned}
$$


Again, recalling that it follows from Proposition 4.5, that for each integer $n$, there is a positive constant $c_{n}$ such that $\left|D^{n} \Lambda(u)\right| \leq c_{n} e^{-\rho u}$ for all $u \geq 0$. In particular, this implies that there is a constant $c_{2}$ such that if $s \in \mathbb{C}$, with $\operatorname{Re} s>0$, then $\left|\Lambda^{\prime \prime}(u) u^{s+1}\right| \leq c_{2} e^{-\rho u} u^{\operatorname{Re} s+1}$. We conclude from this and (6.8) that if $s \in \mathbb{C}$, with $\operatorname{Re} s>0$, then

$$
\begin{aligned}
|Z(s)| & \leq \frac{1}{|s|^{2}} \int_{0}^{\infty}\left|\Lambda^{\prime \prime}(u) u^{s+1}\right| d u \\
& \leq c_{2} \frac{1}{|s|^{2}} \int_{0}^{\infty} e^{-r u} u^{\operatorname{Re} s+1} d u \\
& =c_{2} \frac{1}{r^{\operatorname{Re} s+2}} \frac{1}{|s|^{2}} \int_{0}^{\infty} e^{-v} v^{\operatorname{Re} s+1} d u \\
& =c_{2} \frac{1}{r^{\operatorname{Re} s+2}} \Gamma(\operatorname{Re} s+2) \frac{1}{|s|^{2}} .
\end{aligned}
$$

We deduce from this that if $s \in \mathbb{H}_{d}$, then

$$
|Z(s)| \leq h_{d} \frac{1}{|s|^{2}}
$$

where $h_{d}=c_{2} \sup _{x \in[0, d]}\left(\frac{1}{r^{x+2}} \Gamma(x+2)\right)<\infty$.

(2) Define $f: \mathbb{C} \rightarrow \mathbb{R}$ by $f(s)=1-q r^{-s}$ and write $\mathbb{I}=\left\{z \in \mathbb{C} \mid-\frac{\pi}{-\log r} \leq \operatorname{Im} z \leq\right.$ $\left.\frac{\pi}{-\log r}\right\}$. It is clear that $f$ is periodic with period equal to $\frac{2 \pi}{-\log r}$, and so $\inf _{s \in \overline{\mathbb{K}}_{d}}|f(s)|=$ $\inf _{s \in \overline{\mathbb{K}_{d}} \cap \mathbb{I}}|f(s)|$. It is also clear that $f(s)=0$ if and only if $s \in \Delta+\frac{2 \pi}{-\log r} \mathbb{Z}$. Since $d \neq \Delta$, we deduce from this that $f(s) \neq 0$ for $s \in \overline{\mathbb{K}_{d}} \cap \mathbb{I}$, and the compactness of $\overline{\mathbb{K}_{d}} \cap \mathbb{I}$ therefore shows that there is a real constant $k_{d}$ such that $|f(s)| \geq \frac{1}{k_{d}}$ for all $s \in \overline{\mathbb{K}_{d}} \cap \mathbb{I}$, whence $\inf _{s \in \mathbb{K}_{d}}|f(s)| \geq \inf _{s \in \overline{\mathbb{K}}_{d}}|f(s)|=\inf _{s \in \overline{\mathbb{K}_{d}} \cap \mathbb{I}}|f(s)| \geq \frac{1}{k_{d}}$. This implies that $\frac{1}{|f(s)|} \leq k_{d}$ for all $s \in \mathbb{K}_{d}$.

Below we use the following notation, namely, if $f$ is a holomorphic function, then $P(f)$ denotes the set of poles of $f$, and if $\omega$ is a pole of $f$, then $\operatorname{res}(f ; \omega)$ denotes the residue of $f$ at $\omega$.

Theorem 6.5 For $n \in \mathbb{Z}$, write

$$
s_{n}=\Delta+\frac{1}{-\log r} 2 \pi \dot{\mathrm{i}} n .
$$

Let $u>0$.

(1) We have

$$
P\left(s \rightarrow \frac{Z(s)}{1-q r^{-s}} u^{-s}\right)=\left\{s_{n} \mid n \in \mathbb{Z}\right\} .
$$

(2) For $n \in \mathbb{Z}$, we have

$$
\operatorname{res}\left(s \rightarrow \frac{Z(s)}{1-q r^{-s}} u^{-s} ; s_{n}\right)=\frac{1}{-\log r} Z\left(s_{n}\right) u^{-s_{n}}
$$

(3) We have

$$
\sum_{n \in \mathbb{Z}}\left|\operatorname{res}\left(s \rightarrow \frac{Z(s)}{1-q r^{-s}} u^{-s} ; s_{n}\right)\right|<\infty
$$


In particular, the series

$$
\begin{aligned}
\sum_{n \in \mathbb{Z}} \operatorname{res}\left(s \rightarrow \frac{Z(s)}{1-q r^{-s}} u^{-s} ; s_{n}\right) & =\sum_{n \in \mathbb{Z}} \frac{1}{-\log r} Z\left(s_{n}\right) u^{-s_{n}} \\
& =u^{-\Delta} \frac{1}{-\log r} \sum_{k \in \mathbb{Z}} Z\left(s_{n}\right) e^{2 \pi \dot{\mathrm{i} n} \frac{\log u}{\log r}} \\
& =u^{-\Delta} \Pi(u),
\end{aligned}
$$

where $\Pi:(0, \infty) \rightarrow \mathbb{C}$ is defined by

$$
\Pi(u)=\frac{1}{-\log r} \sum_{k \in \mathbb{Z}} Z\left(s_{n}\right) e^{2 \pi \mathrm{i} i \frac{\log u}{\log r}},
$$

converges. In addition, П is a multiplicatively periodic function with period equal to $r$, i.e. $\Pi(r u)=\Pi(u)$ for all $u>0$.

Proof (1) This statement is clear.

(2) This statement is clear.

(3) Note that it follows from Theorem 6.4 that there is a constant $h_{\Delta}$ such that $|Z(s)| \leq$ $h_{\Delta} \frac{1}{|s|^{2}}$ for all complex numbers $s$ with $0<\operatorname{Re} s \leq \Delta$. In particular, since Re $s_{n}=\Delta$ for all $n \in \mathbb{Z}$, this implies that

$$
\left|Z\left(s_{n}\right)\right| \leq h_{\Delta} \frac{1}{\left|s_{n}\right|^{2}}
$$

for all $n$. We now conclude from this and parts (1) and (2) that

$$
\begin{aligned}
\sum_{n \in \mathbb{Z}}\left|\operatorname{res}\left(s \rightarrow \frac{Z(s)}{1-q r^{-s}} u^{-s} ; s_{n}\right)\right| & =\sum_{n \in \mathbb{Z}}\left|\frac{1}{-\log r} Z\left(s_{n}\right) u^{-s_{n}}\right| \\
& =\frac{1}{-\log r} \sum_{n \in \mathbb{Z}}\left|Z\left(s_{n}\right)\right| \mid u^{-s_{n} \mid} \\
& \leq \frac{h_{\Delta}}{-\log r} \sum_{n \in \mathbb{Z}} \frac{1}{\left|s_{n}\right|^{2}} u^{-\operatorname{Re} s_{n}} \\
& \leq \frac{h_{\Delta}}{-\log r} \sum_{n \in \mathbb{Z}} \frac{1}{\Delta^{2}+\left(\frac{1}{-\log r} 2 \pi\right)^{2} n^{2}} u^{-\Delta} \\
& <\infty .
\end{aligned}
$$

This completes the proof.

Theorem 6.6 Let $\Pi:(0, \infty) \rightarrow \mathbb{C}$ be defined as in Theorem 6.5. For all real numbers $d$ with $\Delta<d$, there is a constant $K_{d}$ such that

$$
\left|\frac{1}{2 \pi \dot{\mathrm{i}}} \int_{c-\mathrm{i} \infty}^{c+\mathrm{i} \infty} \frac{Z(s)}{1-q r^{-s}} u^{-s} d s-u^{-\Delta} \Pi(u)\right| \leq K_{d} \frac{1}{u^{d}}
$$

for all $u>0$ and all real numbers $c$ with $0<c<\Delta$.

In particular, for all real numbers $c$ and $d$ with $0<c<\Delta<d$, we have

$$
\frac{1}{2 \pi \dot{\mathrm{i}}} \int_{c-\mathrm{i} \infty}^{c+\mathrm{i} \infty} \frac{Z(s)}{1-q r^{-s}} u^{-s} d s=u^{-\Delta} \Pi(u)+\mathcal{O}\left(\frac{1}{u^{d}}\right)
$$

for all $u>0$. 
Proof Write $a_{n}=\operatorname{Im}\left(\frac{s_{n}+s_{n-1}}{2}\right)$ for $n \in \mathbb{N}$.

Fix a real numbers $c$ and $d$ with $0<c<\Delta<d$ and let $\Gamma_{d, n}, \gamma_{c, d, n}^{-}$and $\gamma_{c, d, n}^{+}$denote the following paths in $\mathbb{C}$ :

$$
\begin{aligned}
& \Gamma_{d, n} \text { is the directed line segment from } d+\dot{\mathrm{i}} a_{n} \text { to } d-\dot{\mathrm{i}} a_{n} \text {; } \\
& \gamma_{c, d, n}^{-} \text {is the directed line segment from } d-\dot{\mathrm{i}} a_{n} \text { to } c-\dot{\mathrm{i}} a_{n} \text {; } \\
& \gamma_{c, d, n}^{+} \text {is the directed line segment from } c+\dot{\mathrm{i}} a_{n} \text { to } d+\dot{\mathrm{i}} a_{n} \text {. }
\end{aligned}
$$

Fix $u>0$. Let $G_{c, d, n}$ denote the region enclosed by the paths $\Gamma_{d, n}, \gamma_{c, d, n}^{-}, \gamma_{c, d, n}^{+}$and the directed line segment from $c-\dot{\mathrm{i}} a_{n}$ to $c+\dot{\mathrm{i}} a_{n}$. Since $G_{c, d, n} \cap P\left(s \rightarrow \frac{Z(s)}{1-q r^{-s}} u^{-s}\right)=\left\{s_{k}|| k \mid<\right.$ $n\}$, it now follows from the residue theorem applied to the function $s \rightarrow \frac{Z(s)}{1-q r^{-s}} u^{-s}$ where $s \in \mathbb{C}$ with $\operatorname{Re} s>0$ that

$$
\begin{aligned}
& \frac{1}{2 \pi \dot{\mathrm{i}}} \int_{c-\dot{\mathrm{i}} a_{n}}^{c+\mathrm{i} a_{n}} \frac{Z(s)}{1-q r^{-s}} u^{-s} d s+\frac{1}{2 \pi \dot{\mathrm{i}}} \int_{\Gamma_{d, n}} \frac{Z(s)}{1-q r^{-s}} u^{-s} d s \\
& +\frac{1}{2 \pi \dot{\mathrm{i}}} \int_{\gamma_{c, d, n}^{-}} \frac{Z(s)}{1-q r^{-s}} u^{-s} d s+\frac{1}{2 \pi \dot{\mathrm{i}}} \int_{\gamma_{c, d, n}^{+}} \frac{Z(s)}{1-q r^{-s}} u^{-s} d s \\
& \quad=\sum_{|k|<n} \operatorname{res}\left(s \rightarrow \frac{Z(s)}{1-q r^{-s}} u^{-s} ; s_{k}\right)
\end{aligned}
$$

and so

$$
\begin{aligned}
\mid & \frac{1}{2 \pi \dot{\mathrm{i}}} \int_{c-\dot{\mathrm{i}} a_{n}}^{c+\mathrm{i} a_{n}} \frac{Z(s)}{1-q r^{-s}} u^{-s} d s-\sum_{|k|<n} \operatorname{res}\left(s \rightarrow \frac{Z(s)}{1-q r^{-s}} u^{-s} ; s_{k}\right) \mid \\
= & \mid \frac{1}{2 \pi \dot{\mathrm{i}}} \int_{\Gamma_{d, n}} \frac{Z(s)}{1-q r^{-s}} u^{-s} d s \\
& +\frac{1}{2 \pi \dot{\mathrm{i}}} \int_{\gamma_{c, d, n}^{-}} \frac{Z(s)}{1-q r^{-s}} u^{-s} d s+\frac{1}{2 \pi \dot{\mathrm{i}}} \int_{\gamma_{c, d, n}^{+}} \frac{Z(s)}{1-q r^{-s}} u^{-s} d s \mid \\
\leq & \frac{1}{2 \pi}\left|\int_{\Gamma_{d, n}} \frac{Z(s)}{1-q r^{-s}} u^{-s} d s\right| \\
& +\frac{1}{2 \pi}\left|\int_{\gamma_{c, d, n}^{-}} \frac{Z(s)}{1-q r^{-s}} u^{-s} d s\right|+\frac{1}{2 \pi}\left|\int_{\gamma_{c, d, n}^{+}} \frac{Z(s)}{1-q r^{-s}} u^{-s} d s\right| .
\end{aligned}
$$

Next, we note that it follows from Theorem 6.5 and Theorem 6.3 that the series $\sum_{k \in \mathbb{Z}} \operatorname{res}\left(s \rightarrow \frac{Z(s)}{1-q r^{-s}} u^{-s} ; s_{k}\right)=u^{-\Delta} \Pi(u)$ is convergent and that $L(u)=\frac{1}{2 \pi \dot{\mathrm{i}}} \int_{c-\mathrm{i} \infty}^{c+\mathrm{i} \infty}$ $\frac{Z(s)}{1-q r^{-s}} u^{-s} d s$, respectively, whence

$$
\begin{aligned}
& \left|\frac{1}{2 \pi \dot{\mathrm{i}}} \int_{c-\dot{\mathrm{i}} a_{n}}^{c+\dot{\mathrm{i}} a_{n}} \frac{Z(s)}{1-q r^{-s}} u^{-s} d s-u^{-\Delta} \Pi(u)\right| \\
& =\left|\frac{1}{2 \pi \dot{\mathrm{i}}} \int_{c-\dot{\mathrm{i}} \infty}^{c+\dot{\mathrm{i}} \infty} \frac{Z(s)}{1-q r^{-s}} u^{-s} d s-\sum_{k \in \mathbb{Z}} \operatorname{res}\left(s \rightarrow \frac{Z(s)}{1-q r^{-s}} u^{-s} ; s_{k}\right)\right| \\
& \leq v_{c, n}(u)+\left|\frac{1}{2 \pi \dot{\mathrm{i}}} \int_{c+\dot{\mathrm{i}} a_{n}}^{c+\dot{\mathrm{i}} a_{-n}} \frac{Z(s)}{1-q r^{-s}} u^{-s} d s-\sum_{|k|<n} \operatorname{res}\left(s \rightarrow \frac{Z(s)}{1-q r^{-s}} u^{-s} ; s_{k}\right)\right| \\
& \quad+w_{c, n}(u)
\end{aligned}
$$


for all positive integers $n$, where

$$
\begin{aligned}
v_{c, n}(u) & =\left|\frac{1}{2 \pi \dot{\mathrm{i}}} \int_{c-\dot{\mathrm{i}} \infty}^{c+\mathrm{i} \infty} \frac{Z(s)}{1-q r^{-s}} u^{-s} d s-\frac{1}{2 \pi \dot{\mathrm{i}}} \int_{c-\dot{\mathrm{i}} a_{n}}^{c+\mathrm{i} a_{n}} \frac{Z(s)}{1-q r^{-s}} u^{-s} d s\right|, \\
w_{c, n}(u) & =\left|\sum_{|k|<n} \operatorname{res}\left(s \rightarrow \frac{Z(s)}{1-q r^{-s}} u^{-s} ; s_{k}\right)-\sum_{k \in \mathbb{Z}} \operatorname{res}\left(s \rightarrow \frac{Z(s)}{1-q r^{-s}} u^{-s} ; s_{k}\right)\right| .
\end{aligned}
$$

Combining (6.9) and (6.10) we now conclude that

$$
\begin{aligned}
& \left|\frac{1}{2 \pi \dot{\mathrm{i}}} \int_{c-\dot{\mathrm{i}} a_{n}}^{c+\dot{\mathrm{i}} a_{n}} \frac{Z(s)}{1-q r^{-s}} u^{-s} d s-u^{-\Delta} \Pi(u)\right| \leq v_{c, n}(u)+w_{c, n}(u) \\
& \quad+\frac{1}{2 \pi}\left|\int_{\Gamma_{d, n}} \frac{Z(s)}{1-q r^{-s}} u^{-s} d s\right|+\frac{1}{2 \pi}\left|\int_{\gamma_{c, d, n}^{-}} \frac{Z(s)}{1-q r^{-s}} u^{-s} d s\right| \\
& \quad+\frac{1}{2 \pi}\left|\int_{\gamma_{c, d, n}^{+}} \frac{Z(s)}{1-q r^{-s}} u^{-s} d s\right| .
\end{aligned}
$$

We now prove the follows claims.

Claim 1 For all $c$ with $0<c<\Delta$, we have $v_{c, n}(u) \rightarrow 0$ as $n \rightarrow \infty$ for all $u>0$ and $w_{c, n}(u) \rightarrow 0$ as $n \rightarrow \infty$ for all $u>0$.

Proof of Claim 1 This statement follows immediately from the definition of $v_{c, n}(u)$ and $w_{c, n}(u)$. This completes the proof of Claim 1 .

Claim 2 For all $c$ and $d$ with $0<c<\Delta<d$, we have $\left|\int_{\gamma_{c, d, n}^{ \pm}} \frac{Z(s)}{1-q r^{-s}} u^{-s} d s\right| \rightarrow 0$ as $n \rightarrow \infty$ for all $u>0$.

Proof of Claim 2 Let the sets $\mathbb{H}_{d}$ and $\mathbb{K}_{d}$ be defined as in Theorem 6.4 and note that it follows from Theorem 6.4 that there are constants $h_{d}$ and $k_{d}$ such that $|Z(s)| \leq h_{d} \frac{1}{|s|^{2}}$ for all $s \in \mathbb{H}_{d}$ and $\frac{1}{\left|1-q r^{-s}\right|} \leq k_{d}$ for $s \in \mathbb{K}_{d}$. Since $\gamma_{c, d, n}^{ \pm} \subseteq \mathbb{K}_{d} \subseteq \mathbb{H}_{d}$, we therefore conclude that if $u>0$ and $n \in \mathbb{N}$, then

$$
\begin{aligned}
\left|\int_{\gamma_{c, d, n}^{ \pm}} \frac{Z(s)}{1-q r^{-s}} u^{-s} d s\right| & \leq \int_{\gamma_{c, d, n}^{ \pm}} \frac{|Z(s)|}{\left|1-q r^{-s}\right|}\left|u^{-s}\right| d s \\
& \leq h_{d} k_{d} \int_{\gamma_{c, d, n}^{ \pm}} \frac{1}{|s|^{2}} u^{-\operatorname{Re} s} d s \\
& =h_{d} k_{d} \int_{c}^{d} \frac{1}{t^{2}+a_{n}^{2}} u^{-t} d t \\
& \leq h_{d} k_{d} \int_{c}^{d} u^{-t} d t \frac{1}{a_{n}^{2}} \\
& \rightarrow 0 \text { as } n \rightarrow \infty
\end{aligned}
$$

since $a_{n} \rightarrow \infty$ as $n \rightarrow \infty$. This completes the proof of Claim 2 .

Claim 3 For all $d$ with $\Delta<d$, there is a constant $K_{d}$ such that $\left|\int_{\Gamma_{d, n}} \frac{Z(s)}{1-q r^{-s}} u^{-s} d s\right| \leq$ $K_{d} \frac{1}{u^{d}}$ for all $u \geq 0$ and all $n \in \mathbb{N}$. 
Proof of Claim 3 Again, let the sets $\mathbb{H}_{d}$ and $\mathbb{K}_{d}$ be defined as in Theorem 6.4 and, again, note that it follows from Theorem 6.4 that there are constants $h_{d}$ and $k_{d}$ such that $|Z(s)| \leq h_{d} \frac{1^{|s|}}{}{ }^{2}$ for all $s \in \mathbb{H}_{d}$ and $\frac{1}{\mid 1-q r^{-s \mid}} \leq k_{d}$ for $s \in \mathbb{K}_{d}$. Since $\Gamma_{d, n} \subseteq \mathbb{K}_{d} \subseteq \mathbb{H}_{d}$, we therefore conclude that if $u>0$ and $n \in \mathbb{N}$, then

$$
\begin{aligned}
\left|\int_{\Gamma_{d, n}} \frac{Z(s)}{1-q r^{-s}} u^{-s} d s\right| & \leq \int_{\Gamma_{d, n}} \frac{|Z(s)|}{\left|1-q r^{-s}\right|}\left|u^{-s}\right| d s \\
& \leq h_{d} k_{d} \int_{\Gamma_{d, n}} \frac{1}{|s|^{2}} u^{-\operatorname{Re} s} d s \\
& =h_{d} k_{d} \int_{\Gamma_{d, n}} \frac{1}{|s|^{2}} u^{-d} d s \\
& =h_{d} k_{d} u^{-d} \int_{-a_{n}}^{a_{n}} \frac{1}{d^{2}+t^{2}} d t \\
& \leq h_{d} k_{d} u^{-d} \int_{-\infty}^{\infty} \frac{1}{d^{2}+t^{2}} d t \\
& =K_{d} u^{-d}
\end{aligned}
$$

where $K_{d}=h_{d} k_{d} \int_{-\infty}^{\infty} \frac{1}{d^{2}+t^{2}} d t$. This completes the proof of Claim 3 .

Combining (6.11) and Claim 3 we conclude that

$$
\begin{aligned}
& \left|\frac{1}{2 \pi \dot{\mathrm{i}}} \int_{c-\dot{\mathrm{i}} a_{n}}^{c+\dot{\mathrm{a}} a_{n}} \frac{Z(s)}{1-q r^{-s}} u^{-s} d s-u^{-\Delta} \Pi(u)\right| \leq v_{c, n}(u)+w_{c, n}(u) \\
& +\frac{1}{2 \pi}\left|\int_{\Gamma_{d, n}} \frac{Z(s)}{1-q r^{-s}} u^{-s} d s\right|+\frac{1}{2 \pi}\left|\int_{\gamma_{c, d, n}^{-}} \frac{Z(s)}{1-q r^{-s}} u^{-s} d s\right| \\
& +\frac{1}{2 \pi}\left|\int_{\gamma_{c, d, n}^{+}} \frac{Z(s)}{1-q r^{-s}} u^{-s} d s\right| \leq v_{c, n}(u)+w_{c, n}(u) \\
& +\frac{1}{2 \pi}\left|\int_{\gamma_{c, d, n}^{-}} \frac{Z(s)}{1-q r^{-s}} u^{-s} d s\right|+\frac{1}{2 \pi}\left|\int_{\gamma_{c, d, n}^{+}} \frac{Z(s)}{1-q r^{-s}} u^{-s} d s\right|+K_{d} u^{-d}
\end{aligned}
$$

for all $u>0$ and all $n$. Letting $n \rightarrow \infty$ and using Claims 1 and 2, we deduce from this inequality that

$$
\left|\frac{1}{2 \pi \dot{\mathrm{i}}} \int_{c-\dot{\mathrm{i} \infty} \infty}^{c+\mathrm{i} \infty} \frac{Z(s)}{1-q r^{-s}} u^{-s} d s-u^{-\Delta} \Pi(u)\right| \leq K_{d} u^{-d}
$$

for all $u>0$.

We can now prove inequality (6.1).

Theorem 6.7 Let $\Pi:(0, \infty) \rightarrow \mathbb{C}$ be defined as in Theorem 6.5. For all real numbers $d$ with $\Delta<d$, there is a constant $K_{d}$ such that

$$
\left|L(u)-u^{-\Delta} \Pi(u)\right| \leq K_{d} \frac{1}{u^{d}}
$$

for all $u>0$.

Proof This follows immediately by combining Theorems 6.3 and 6.6. 


\section{Proof of Theorems 2.4 and 2.5}

Finally, we will now combine inequalities (2.7) and (2.8) (i.e. Theorems 5.3 and 6.7, respectively), to prove Theorems 2.4 and 2.5 .

Theorem 2.4 and Theorem 2.5 Let $\Pi:(0, \infty) \rightarrow \mathbb{C}$ be defined as in Theorem 6.5. Then there is a sequence $\left(\varepsilon_{n}\right)_{n}$ with $\lim \sup _{n} n\left|\varepsilon_{n}\right|<\infty$ such that

$$
n^{\Delta} M_{n}=\Pi(n)+\varepsilon_{n}
$$

for all $n$.

Proof It follows from Theorem 5.3 that there is a constant $K$ such that $\left|M_{n}-L(n)\right| \leq K \frac{1}{n^{1+\Delta}}$ for all $n$, and it follows from Theorem 6.7 that for each $d$ with $\Delta<d$ there is a constant $K_{d}$ such that $\left|L(u)-n^{-\Delta} \Pi(u)\right| \leq K_{d} \frac{1}{u^{d}}$ for all $u>0$. Hence

$$
\left|M_{n}-n^{-\Delta} \Pi(n)\right| \leq\left|M_{n}-L(n)\right|+\left|L(n)-n^{-\Delta} \Pi(n)\right| \leq K \frac{1}{n^{1+\Delta}}+K_{d} \frac{1}{u^{d}}
$$

for all $d$ with $\Delta<d$ and all $n$. In particular, for $d=1+\Delta$, we have

$$
\left|M_{n}-n^{-\Delta} \Pi(n)\right| \leq K \frac{1}{n^{1+\Delta}}+K_{1+\Delta} \frac{1}{n^{1+\Delta}}=\left(K+K_{1+\Delta}\right) \frac{1}{n^{1+\Delta}}
$$

for all $n$. This clearly implies the desired result.

Acknowledgements The authors thank an anonymous referee for providing the references [4,5,11] and for pointing out the connection between this work and recent work on the Kantorovich-Wasserstein distance between self-similar measures in $[4,5,11]$.

Open Access This article is distributed under the terms of the Creative Commons Attribution 4.0 International License (http://creativecommons.org/licenses/by/4.0/), which permits unrestricted use, distribution, and reproduction in any medium, provided you give appropriate credit to the original author(s) and the source, provide a link to the Creative Commons license, and indicate if changes were made.

\section{References}

1. Bailey, D.H., Borwein, J.M., Crandall, R.E., Rose, M.G.: Expectations on fractal sets. Appl. Math. Comput. 220, 695-721 (2013)

2. Bandt, C., Kuschel, T.: Self-similar sets. VIII. Average interior distance in some fractals. Measure theory (Oberwolfach, 1990). Rend. Circ. Mat. Palermo (2) Suppl 28, 307-317 (1992)

3. Billingsley, P.: Convergence of Probability Measures. Wiley, New York (1968)

4. Cipriano, I., Pollicott, M.: Stationary measures associated to analytic iterated function schemes. Preprint (2015)

5. Cipriano, I.: The Wasserstein distance between stationary measures associated to iterated function schemes on the unit interval. Preprint (2016) arXiv:1611.00092

6. Cristea, L., Prodinger, H.: Moments of distributions related to digital expansions. J. Math. Anal. Appl. 315, 606-625 (2006)

7. Escribano, C., Sastre, M., Torrano, E.: Moments of infinite convolutions of symmetric Bernoulli distributions. J. Comput. Appl. Math. 153, 191-199 (2003)

8. Escribano, C., Sastre, M., Torrano, E.: Moment matrix of a self-similar measure. Electr. Trans. Numer. Anal. 4, 79-87 (2006)

9. Falconer, K.J.: Fractal Geometry: Mathematical Foundations and Applications. Wiley, Chichester (1990)

10. Falconer, K.J.: Techniques in Fractal Geometry. Wiley, New York (1997)

11. Fraser, J.: First and second moments for self-similar couplings and Wasserstein distances. Math. Nachr. 288, 2028-2041 (2015) 
12. Goh, W., Wimp, J.: Asymptotics for the moments of singular distributions. J. Approx. Theory 74, 301-334 (1993)

13. Grabner, P., Prodinger, H.: Asymptotic analysis of the moments of the Cantor distribution. Stat. Probab. Lett. 26, 243-248 (1996)

14. Hinz, A.M., Schief, A.: The average distance on the Sierpinski gasket. Probab. Theory Relat. Fields 87, 129-138 (1990)

15. Jacquet, P., Szpankowski, W.: Analytical depoissonization and its applications. Theor. Comput. Sci. 201, $1-62(1998)$

16. Lapidus, M.L., van Frankenhuysen, M.: Fractal Geometry and Number Theory: Complex Dimensions of Fractal Strings and Zeros of Zeta Functions. Birkhäuser, Boston (2000)

17. Lapidus, M.L., van Frankenhuysen, M.: Fractal Geometry, Complex Dimensions and Zeta Functions: Geometry and Spectra of Fractal Strings. Springer Monographs in Mathematics. Springer, New York (2006)

18. Leary, C.C., Ruppe, D.A., Hartvigsen, G.: Fractals, average distance and the Cantor set. Fractals 18, 327-341 (2010)

19. Patterson, S.J.: An Introduction to the Theory of the Riemann Zeta-Function. Cambridge Studies in Advanced Mathematics, vol. 14. Cambridge University Press, Cambridge (1988)

20. Villani, C.: Optimal Transport. Springer, Berlin (2009) 